\title{
NOTAS SOBRE EL VIAJE DE LA FRAGATA \\ BALLENERA EMELIA A LOS MARES DEL SUR (1788-1790) \\ EL INFORME DEL CAPITÁN JAMES SHIELDS A ENDERBY \& SONS Y OTROS DOCUMENTOS
}

DANIEL QUIROZ

\begin{abstract}
RESUMEN
En este trabajo se escriben algunas notas sobre el viaje de un grupo de balleneros ingleses que entre 1788 y 1790 se dirigió a cazar cachalotes al océano Pacífico a bordo del Emelia, el primer buque en cruzar el cabo de Hornos con este propósito. Las notas abarcan una serie de temas que, en conjunto, pretenden entregar una mirada sobre el contexto en el que este viaje se produce y los resultados obtenidos. Incluye una breve descripción del buque, de sus propietarios y también de su capitán. Se pudo reconstruir su itinerario, basado en la carta del capitán Shields a Samuel Enderby \& Sons, propietarios del buque, y un informe enviado a las autoridades británicas por un grupo de comerciantes balleneros. Se entregan algunos datos sobre los viajes que siguen las huellas del Emelia en 1790. Se discute, además, la identidad del arponero que mató el primer cachalote en los mares del sur, supuestamente el primer oficial Archaelus Hammond, de Nantucket.
\end{abstract}

PALABRAS CLAVE: caza de ballenas, balleneros ingleses, océano Pacífico, capitán James Shields, Enderby \& Sons.

NOTES ON THE WHALING SHIP EMELIA'S

JOURNEY TO THE SOUTH SEAS (1788-1790)

CAPTAIN JAMES SHIELDS' REPORT TO ENDERBY \& SONS AND OTHER DOCUMENTS

\section{ABSTRACT}

In this paper some notes are written about the journey of a group of English whalers between 1788 and 1790 to hunt sperm whales in the Pacific Ocean aboard Emelia, the first ship to cross Cape Horn for this purpose. The notes cover a series of topics that, together, aim to provide a look at the context in which this journey takes place and the results obtained. It includes a brief description of the ship, its owners and also its captain. His itinerary could be reconstructed, based on the letter from Captain Shields to Samuel Enderby \& Sons, owners of the ship, and a report sent to the British authorities by a group of whaling traders. Some information is given on the trips

a Subdirección de Investigación, Servicio Nacional del Patrimonio Cultural, Moneda 650, 3er Piso, Santiago, Chile.

\daniel.quiroz@patrimoniocultural.gob.cl 
that follow in the footsteps of Emelia in 1790. In addition, the identity of the harpooner who killed the first sperm whale in the southern seas, allegedly the first officer Archaelus Hammond, from Nantucket is discussed.

KEY WORDS: Whaling, British whalers, Pacific Ocean, Captain James Shields, Enderby \& Sons.

En 1788 una hermosa nave, el Amelia, equipada para este expreso propósito [la caza de cachalotes] y solo a cuenta de los vigorosos Enderbys, dio temerariamente la vuelta al cabo de Hornos y fue la primera entre las naciones en bajar un bote ballenero de cualquier tipo en los mares del Sur.

La travesía fue hábil y afortunada y al volver a su muelle con la bodega llena de la preciosa esperma, el ejemplo del Amelia fue pronto seguido por otros barcos, ingleses y estadounidenses y así se fueron abriendo los vastos caladeros de cachalotes del Pacífico

Herman Melville, Moby Dick, 2002 [1851], p. 341

Samuel Enderby, comerciante de Londres, fue el fundador de la famosa compañia ballenera Enderby \& Sons, una casa que en mi humilde opinión de ballenero, no es menos, en cuanto a real interés histórico, que las casas reales de los Tudor y los Borbones; mis numerosos documentos pesqueros no aclaran desde cuando antes del año 1775 de Nuestro Señor existía esta casa ballenera, pero ese año se equiparon los primeros barcos ingleses que cazaron en forma regular cachalotes.

Herman Melville, Moby Dick, 2002 [1851], pp. 340-341

\section{PRÓLOGO}

Eugenio Pereira Salas, al que podríamos llamar "el padre" de los estudios históricos sobre la caza de ballenas en las costas de Chile, escribe que el viaje ballenero de la fragata Emilia en 1788 , el primero en cazar cachalotes en los caladeros del océano Pacífico, corresponde a una empresa de carácter metódico y deliberado, desarrollada por la firma británica de Samuel Enderby \& Sons, pioneros de esta rama comercial y cuyo éxito se debe a la pericia del capitán Shields y al contramaestre Archaelus Hammond, cuyo nombre envuelto en la leyenda pasó a ser la enseña del valor constructivo (Pereira Salas, 1971, pp. 40-41). El regreso en marzo de 1790 del buque a Londres fue triunfal y trascendente; su cargamento de 139 toneladas del rico espermaceti, demostraba la veracidad promisoria de los relatos del capitán Cook y de los viajeros (Pereira Salas, 1971, p. 41). En sus palabras y transcribiendo las de otros, el proyecto excedió las esperanzas de los armadores, 'en cerca de dieciocho meses volvió repleta de aceite, lo que muestra la abundancia de cachalotes'; el Pacífico iba a entregar las riquezas ictiológicas de su inmensa extensión oceánica; la noticia se expandió con el sigilo misterioso de las novedades comerciales. Los centros de pesquería comenzaron a preparar sus flotillas (Pereira Salas, 1971, pp. 41-42).

El 28 de octubre de 1790, se firma la denominada Convención de Nootka donde España le reconoce a Inglaterra no sólo su derecho a la pesca de ballenas en el Pacífico, sino a residir de forma temporal en los lugares que no estuvieran ocupados por los españoles y a establecer comercio con los naturales del lugar. El artículo III de la Convención dice textualmente: Y a fin de estrechar los vínculos de amistad y de conservar en lo venidero una perfecta harmonía y buena inteligencia entre las dos Partes Contratantes, se ha convenido que los súbditos respectivos no serán perturbados ni molestados, ya sea navegando o pescando en 
el Océano Pacífico ó en los mares del Sur; ya sea desembarcando en las costas que circundan estos mares, en parajes no ocupados ya, a fin de comerciar con los naturales del país, o para formar establecimientos, aunque todo ha de ser con sujeción a las restricciones y providencias que se especificarán en los tres artículos siguientes (Convención de Nootka, 1796, pp. 369-370). Pero a pesar de las restricciones establecidas para dificultar la recalada de buques extranjeros en las costas ocupadas por los españoles, la presencia de naves balleneras de diversas nacionalidades registra un aumento considerable entre 1791 y 1810 , pasando a ser una incómoda rutina en las costas de Chile (Pereira Salas, 1971, p. 69).

Desde ese año, 1790, marcado por la llegada del Emelia a Londres y la firma de la Convención de Nootka, centenares de buques balleneros, ingleses, franceses y estadounidenses, cruzarán el cabo de Hornos en busca de los "preciosos" cachalotes que ofrecen los caladeros del océano Pacífico. El paso continuo de buques por el cabo de Hornos y el estrecho de Magallanes generará un mayor conocimiento de la tierra y el mar patagónico, de sus habitantes.

La travesía del Emelia marca la apertura de un ciclo ballenero audaz y renovador (Pereira Salas, 1971, p. 43). Este trabajo es un modesto homenaje a su sugerente libro, escrito hace ya medio siglo, que abre los estudios sobre las operaciones cinegéticas en las costas del Pacífico suroriental.

\section{LA SOUTHERN WHALE FISHERY}

En el mes de enero de 1789 la fragata ballenera inglesa Emelia cruza, de este a oeste, el cabo de Hornos, transformándose en la primera nave en buscar cachalotes en los caladeros del Pacífico. Su viaje, muy exitoso, tendrá como consecuencia un flujo constante de buques hacia los mares del sur (Jackson, 1978).

Frederick Bennett, cirujano del buque ballenero británico Tuscan, al mando del capitán Stevers, escribe en 1840, después de regresar de una vuelta al mundo realizada entre 1833 y 1836 , una pequeña, pero precisa nota sobre el viaje de la fragata ballenera Emelia: En el año 1788, el buque de los Srs. Enderby, el Emilia, rodea el Cabo de Hornos y es el primero en cazar cachalotes en el Océano Pacífico (Bennett, 1840, II, p. 185).

Thomas Beale, otro cirujano de buque ballenero, del Kent, capitán Laughton, ofrece, en su conocida obra sobre la biología de los cachalotes y su aprovechamiento por las poblaciones humanas, una descripción notable de la relevancia histórica de la travesía:

En 1788 la gran especulación mercantil que permitió enviar barcos al Pacífico rodeando el Cabo de Hornos para extender la pesca de ballenas de esperma, estuvo reservada para la mente audaz y emprendedora del Sr. Enderby, un comerciante y armador de Londres, que preparó con un gran gasto el buque Amelia, capitán Shields, que zarpó de Inglaterra el $1^{\circ}$ de septiembre de 1788 y regresó el 12 de marzo de 1790, después de una ausencia de un año y siete meses, pero llevando a casa un enorme cargamento de 139 toneladas de aceite de esperma, y tuvo, además, la buena fortuna de recibir 800 libras como prima debido a la naturaleza particular de la expedición; el Amelia había sido el primer barco, de cualquier país, que había entrado al Pacífico en busca de ballenas, su éxito le dio un extraordinario impulso a todas las personas comprometidas en las pesquerías, de modo que varios buques, tanto de este pais [Inglaterra] como de Estados Unidos, inmediatamente siguieron sus pasos (Beale, 1839, p. 148).

Se ha señalado que el libro de Beale influirá poderosamente en el Moby Dick de Herman Melville (Shore, 2012).

Se debe entender el viaje del Emelia como parte del desarrollo de la Southern Whale Fishery, pesquería que inicia sus actividades en 1775 con la decidida participación de comerciantes y balleneros de Nantucket que estaban pescando en el océano entre Cabo Verde y Brazil, entre los $31^{\circ}$ y $36^{\circ}$ Sur, y que se trasladaron a Londres como consecuencia de la Guerra Revolucionaria [17751783] (Jackson, 1978, p. 92). Los británicos 
llamaron Southern Whale Fishery [SWF] a toda la caza de ballenas realizada al sur del paralelo $44^{\circ}$ Norte para diferenciarla de la Northern Whale Fishery [NWF], que se desarrollaba al norte de ese paralelo (Jackson, 1978).

La SWF cuenta desde sus inicios con la iniciativa y entusiasmo de empresarios londinenses y el decidido apoyo de los círculos gubernamentales, quienes apreciaban el valor de las materias primas para una nación que se estaba industrializando, recalcando que el interés en el aceite era el interés de la nación (Jackson, 1978, p. 93). Fue establecida formalmente por el acta 16 Geo. III, c. 47, de 1776, donde, luego de reconocer la valiosa pesquería de ballenas descubierta en los mares al sur de la latitud 44 grados norte, garantiza que los buques involucrados en ella reciban las mismas recompensas que los que trabajaban al norte de ese paralelo (Holt, 1820, p. 204). El 16 de junio de 1786 se establece el acta 26 Geo. III, c. 50 (Act for the Encouragement of the Southern Whale Fishery) que buscaba promover y delimitar las actividades balleneras británicas al este del cabo Buena Esperanza y al oeste del cabo de Hornos. El artículo XV del mencionado documento indica que cualquier buque o velero que navegue o pase al oeste del cabo de Hornos o a través del estrecho de Magallanes para el propósito mencionado [SWF] no deberá pasar al norte de la línea equinoccial o hacer más de 50 grados de longitud Oeste del Cabo de Hornos (Russell, 1786, p. 340). Es interesante resaltar que se premiaba a los buques que fueran más allá de los $36^{\circ} \mathrm{S}$ y regresaran entre 18 y 28 meses después del $1^{\circ}$ de mayo del año de su partida (Jackson, 1978, p. 101). Esta acta fue modificada posteriormente, en varias oportunidades, con el principal propósito de extender el área permitida de captura de ballenas en el océano Pacífico, tanto hacia el norte como el oeste (Clayton, 2014).

1 An account of the Number of Ships, and their Tonnage, fitted out in Great Britain for the Southern Whale Fishery, April 10, 1786. Public Record Office, PRO 30/8/259, f. 199. National Archives, Kew, Inglaterra.

2 Commissioners Customers on Memorial of Samuel Enderby \& Premium on 5 ships employed in the Southern Whale Fishery. Records of Treasury, T1/671, item 1566. National Archives, Kew, Inglaterra.

3 A State of the Southern Whale Fishery, May 15, 1790.
En 1776 participaron de la SWF 12 buques con un arqueo de 1.977 toneladas. En 1784 fueron 15, con 3.109 toneladas. El número varió mucho durante los años intermedios: 13 [2.103 ton] en 1777, 19 [3.038 ton] en 1778, 4 [467 ton] en 1779, 7 [771 ton] en 1780, 6 [757 ton] en 1781, 6 [910 ton] en 1782 , y 9 [1.040 ton] en $1783^{1}$.

El uso de incentivos para empresarios privados en aquellas áreas de la economía que eran de interés gubernamental era una práctica bastante común y muy extendida. Para cobrar los premios ofrecidos por las autoridades, los empresarios balleneros debían enviar a la Oficina de Aduanas la documentación necesaria que comprobara el cumplimiento de los distintos requisitos para obtenerlos y contar con la aprobación mediante un informe elaborado y firmado por un Comisionado de Aduanas ${ }^{2}$.

En un cuadro comparativo elaborado en 1790 por la Aduana de Londres (Tabla 1) ${ }^{3}$, se muestra que entre 1785 y 1789 llegó de la SWF un promedio anual de 28 buques, distribuidos de la siguiente manera: 11 en 1785, 16 en 1786, 28 en 1787, 42 en 1788 y 42 en 1789. La productividad de la flota alcanzó las 10.005 toneladas de aceite (promedio anual de 2.000 toneladas), con una ganancia, considerando no sólo el aceite, sino también las barbas de ballena y las pieles de lobo marino, de $£ 270.382$ (promedio anual de $£ 54.076)^{4}$.

Existen algunas discrepancias entre los datos entregados por los empresarios balleneros y los datos oficiales proporcionados por la Aduana de Londres. Según lo manifestado por Enderby \& Sons, el año 1788 habían regresado 45 de los buques empleados en la SWF, que trajeron en total 668 toneladas de aceite de esperma, 2.424 toneladas de aceite de ballena, 1.817 quintales de barbas de ballena y 64.857 pieles

Public Record Office, PRO 30/8/259, f. 161. National Archives, Kew, Inglaterra.

4 Si tomamos en cuenta el precio real de una mercancía (aceite, barbas de ballena, pieles) y la inflación estimada para el período 1790-2019 [https://www.bankofengland. co.uk/monetary-policy/inflation/inflation-calculator], $£ 1$ de 1790 sería equivalente a $£ 152$ de 2019. Es decir, las ganancias de la SWF para el período 1785-1789 fueron de $£ 41.098 .064$ actuales. 
Tabla 1. Estado de la Southern Whale Fishery entre 1785 y 1789.

\begin{tabular}{ccccccccc}
\hline Año & Naves & Q aceite & V aceite & Q barbas & V barbas & Q pieles & V pieles & V total \\
\hline & $\mathrm{N}$ & ton & $£$ & cwt & $£$ & $\mathrm{~N}$ & $£$ & $£$ \\
1785 & 11 & 696 & 14.418 & 37 & 186 & 0 & 0 & 14.604 \\
1786 & 16 & 1.113 & 25.599 & 357 & 1.787 & 772 & 77 & 27.463 \\
1787 & 28 & 2.181 & 50.182 & 1.389 & 6.947 & 14.280 & 1.428 & 58.557 \\
1788 & 42 & 2.604 & 59.910 & 1.387 & 6.936 & 53.303 & 5.330 & 72.176 \\
1789 & 42 & 3.411 & 78.536 & 2.369 & 11.845 & 72.010 & 7.201 & 97.582 \\
\hline Total & 139 & 10.005 & 228.645 & 5.539 & 27.701 & 140.365 & 14.036 & 270.382 \\
\hline
\end{tabular}

Fuente: Custom Office (1790). A State of Southern Whale Fishery, May 16, 1790. Public Record Office 30/8/259. National Archives, Kew, Londres, Inglaterra.

$$
\begin{aligned}
& \text { Simbología: } \\
& \mathrm{Q}=\text { cantidad } \\
& \mathrm{V}=\text { valor } \\
& \mathrm{N}=\text { número } \\
& \text { ton }=\text { toneladas } \\
& \mathrm{cwt}=\text { quintales }
\end{aligned}
$$

de lobo marino. La carga total fue avaluada en $£ 90.599$ y los premios obtenidos por los buques en $£ 6.300$. En este informe se reportan también los precios de cada producto: una tonelada de aceite de esperma costaba $£ 60$, una tonelada de espermaceti [head matter] £68, una tonelada de aceite de ballena $£ 14$, un quintal de barbas de ballena £5 y cada piel de lobo marino £0,1. En diciembre de 1788 había 49 buques británicos ocupados en la SWF pero solamente uno, el Emilia, 278 toneladas, capitán James Shields, de Enderby \& Sons, Londres, "se había dirigido al oeste del Cabo de Hornos" 5 . En noviembre de 1789 había 51 buques ingleses operando en los mares del sur, de los cuales sólo seis habían salido de Gran Bretaña en 1788: el Emilia seguía siendo el único buque en aventurarse "más allá" del cabo de Hornos ${ }^{6}$.

Los principales empresarios comprometidos en esta "pesquería" y cuyos buques regresaron durante 1788 eran Enderby \& Sons, con ocho

5 An account of the vessels now out on the Southern Whale Fishing made up to $31^{\text {st }}$ December 1788. British Library, Liverpool Papers, Volumen I (Add MS 38391 [1788-1789), fs. 92-93.

6 Caledonian Mercury (Edimburgo), 21 de agosto de 1790. naves; Lucas \& Co., con cinco; Champion \& Co., con cuatro, y Mather \& Co., con tres. El buque que más dinero produjo fue el Kent, de Enderby \& Sons, con $£ 5.423^{7}$.

\section{SAMUEL ENDERBY \& SONS}

La historia de la firma Samuel Enderby \& Sons, de Londres, tiene algunos enigmas y malentendidos (Jones, 1991; Payton, 2018). Se sabe que en 1773 Samuel Enderby Sr. ya estaba involucrado, de alguna manera, en el negocio ballenero, ya sea como propietario de buques registrados, tanto en Boston como Londres, que llevaban mercancías a las colonias americanas y traían aceite de ballena a Inglaterra (Fraser, 2014) como de otros buques que operaban en la caza de cetáceos en Groenlandia (Payton, 2018). Su interés en la actividad le habría llegado por su asociación con Charles Buxton, padre de su esposa, comerciante londinense que tenía varios

An account of Number of Ships with their Cargoes, which returns from the Southern Whale Fishery to England in the year 1788, as furnished by Mr. Enderby, one of the Adventurers. Public Record Office, PRO 30/8/259, f. 160. National Archives, Kew, Londres. 
negocios con empresarios de Nantucket, los que le permitieron vincularse con su principal socio americano Thomas Weathley [...] cuyo hijo Nathaniel llevó la mayoria de los negocios de la flota ballenera de Enderby. Nathaniel Weathley, que vivía en Providence, Rhode Island, se casa con una de las hijas de Samuel Enderby, Mary, en 1773 (Payton, 2018, p. 194).

Samuel Enderby Sr. tenía una flota ballenera operativa en 1765 en los Estados Unidos, no sabemos su magnitud. Debido al deterioro de las relaciones entre Inglaterra y sus colonias americanas, Enderby elabora un plan para llevar su flota a Londres: entre 1774 y 1775 vende todos sus buques americanos o registrados ahi a su yerno americano Nathaniel Weathley, [... quien] envía algunos a Londres, y estos, junto a otros, constituirá la primera flota de Enderby en Inglaterra (Payton, 2018, pp. 196197). Entre los buques enviados por Weathley a Londres estaban el Pitt y el Rockingham, ambos usados en 1775 por Enderby en sus primeras campañas balleneras a los caladeros de Brasil (Clayton, 2014). Ese año, una cierta cantidad de buques, la mayoría de construcción americana, fueron preparados para la caza de ballenas bajo el modelo estadounidense por un conjunto de firmas inglesas, las de Champion, Enderby, Buxton y Mather, siendo la mayoría de los capitanes, oficiales y arponeros de Nantucket (Mawer, 1999, p. 36). Para los Enderby, la caza de ballenas en los mares del sur llegó a ser una magnífica obsesión que absorvió la ambición, energía y fortuna de tres generaciones (Mawer, 1999, pp. 36-37).

Samuel Enderby Sr. crea en 1776 , con sus hijos, la sociedad Samuel Enderby \& Sons y junto con otros comerciantes ingleses, envía un total de doce buques a la caza de ballenas en "los mares del sur", hecho que se señala como el punto de partida, la inauguración, de la South Whale Fishery (Stackpole, 1972; Clayton, 2014). La flota regresa con 439 toneladas de aceite, lo que es considerado un notable éxito (Jackson, 1978, p. 92). En 1777 serán 13 buques y 19 en 1778 (Jackson, 1978).

8 An account of Number of Ships with their Cargoes, which returns from the Southern Whale Fishery to England in the year 1788, as furnished by Mr. Enderby, one of the
La firma de Enderby \& Sons fue muy importante en el desarrollo de la Southern Whale Fishery, pero no podemos olvidar que hubo más de trescientos propietarios, grandes y pequeños, involucrados en ella (Jones, 1981a). Sin embargo, para muchos, Samuel Enderby Sr. fue el notable empresario fundador de la industria ballenera londinense en los mares del sur (Clayton, 2014, p. 185), sobre todo porque inaugura en 1775 la caza británica de cachalotes. Si entendemos que la SWF se extiende entre 1775 y 1860, la familia Enderby fue pre-eminente sobre todo en la primera mitad de sus operaciones (Clayton, 2014, p. 188). En la segunda mitad fue perdiendo liderazgo, siendo superada por otros armadores.

De los doce buques involucrados en los inicios de la SWF en 1776, tres pertenecían a Enderby \& Sons: Experiment, 180 ton, capitán Goldsmith; Neptune, 140 ton, capitán Macy; y Rockingham, 200 ton, capitán Clarke. Este hecho lo convertía en el propietario líder en los inicios del comercio (Jones, 1981b, p. 94). La flota de los Enderby crecerá de manera sostenida. En 1788 regresan a Londres ocho de los barcos pertenecientes a Enderby \& Sons que estaban involucrados en la caza de ballenas en los mares del sur: Kent, capitán Pease, 265 ton; Rasper, capitán Gage, 175 ton; Friendship, capitán Goldsmith, 230 ton; Greenwich, capitán Locke, 256 ton; Sandwich, capitán Shields, 195 ton; Atlantic, capitán Délano, 222 ton; Swift, capitán Hales, 189 ton; y British Queen, capitán Paul, 309 ton. La flota obtuvo en total 811 toneladas de aceite de ballena, 533 quintales de barbas de ballena y 7.857 pieles de lobo fino, con un valor total de $£ 18.250$. Ese mismo año salen de Londres seis naves de Enderby \& Sons: Kent, Greenwich, Friendship, Sandwich y Swift, a los que se le agrega el Emelia, capitán Shields, 278 ton, que habría ido al oeste del Cabo de Hornos ${ }^{8}$.

Como bien lo señala Samuel Enderby Jr., en una nota enviada el 30 de agosto de 1790 a William Pitt Jr., Primer Ministro de Gran Bretaña:

La Southern Whale Fishery comenzó desde Inglaterra en el año 1775, cuando

Adventurers. Public Record Office, PRO 30/8/259, f. 160. National Archives, Kew, Londres. 
se emplearon apenas diez buques; su número fluctuó luego como consecuencia de las guerras \& capturas, incluso el año 1782 sólo se usaron tres; desde ese periodo su número ha aumentado anualmente y con mucho éxito; ahora se usan 68 buques con un valor superior a las $£ 200.000$, en los que navegan 1.400 marineros y aprendices, quiénes, más allá de toda duda, proporcionarán los mejores hombres de mar que luego tenga este país ${ }^{9}$.

Charles Enderby, otro de sus hijos, indica medio siglo después, que la SWF había sido establecida por su padre, desarrollada previamente en forma extensiva mediante agentes en los Estados Unidos de América, en ese entonces dependencias británicas; posteriormente pudo abrir al mundo la más notable de estas pesquerías: la pesquería del Océano Pacífico, por el Capitán Shields, del Emilia (Enderby, 1848, p. 6).

\section{LA FRAGATA EMELIA Y EL CAPITÁN JAMES SHIELDS}

La fragata Emelia había sido construida en 1782, en Bristol, Inglaterra. Era un velero de proa redondeada, rechoncho, con tres mástiles, adaptado especialmente para el procesamiento de cachalotes con un horno en medio de la cubierta; [su diseño] establece el modelo [que seguirán] por medio siglo todos los cazadores de cachalotes (Sanderson, 1993, p. 234). Eugenio Pereira Salas agrega que estaba equipada con una moderna quilla de cobre y su silueta marinera de navio de un solo puente se individualizaba por su ancha y respetable eslora (1971, p. 41).

9 Samuel Enderby Jr. a William Pitt Jr. [Statement on Southern Whale Fishery], 30 de agosto de 1790. Public Record Office, PRO 30/8/133, fs. 35-36. National Archives, Kew, Inglaterra.

10 Register of Ships, 1782. En https://babel.hathitrust.org/ $\mathrm{cgi} / \mathrm{pt}$ ?id=hvd.32044019372358\&view=1 up\&seq=98, consultado el 26 de abril de 2020.

11 Register of Ships, 1783. En https://babel.hathitrust.org/ $\mathrm{cgi} / \mathrm{pt}$ ?id=hvd.32044020166674\&view=1 up\&seq=99, consultado el 26 de abril de 2020.

12 Register of Ships, 1786. En https://babel.hathitrust.org/
El buque es registrado por primera vez en 1782 en Bristol, con 300 toneladas; sus propietarios eran Baillie \& Co., su capitán James Fraser, y estaba dedicado al tráfico de esclavos ${ }^{10}$. En 1783 aparece registrado en Liverpool, ahora bajo el nombre de Emilia, con los mismos propietarios y el mismo capitán ${ }^{11}$. En 1786 se registra otra vez en Bristol ${ }^{12}$. El buque fue ocupado entre 1782 y 1788 en el comercio de esclavos entre los puertos ingleses de Bristol y Liverpool con África y América (Richardson, 1997), realizando entre esas fechas cinco viajes, todos al mando del capitán James Fraser (Rediker, 2007). El puerto de extracción siempre fue Bonny, en Nigeria, y los africanos eran llevados a Jamaica, Dominica, Caribe Británico o Indias Occidentales (Richardson, 1997).

El buque es adquirido en 1788 por Samuel Enderby \& Sons, reinscrito en Londres, ahora de 278 toneladas y con James Shields como su capitán; fue adaptado para la caza de ballenas en los mares del sur ${ }^{13}$. Luego de su primera incursión en el océano Pacífico entre 1788 y 1790 , tema de este trabajo, realiza tres nuevos cruceros balleneros a los mismos caladeros, todos con cargo a Enderby \& Sons: 1790-1791, capitán Shields; 1792-1794, capitán Shields; 1794-1796, capitán Quested ${ }^{14}$.

En 1796 el buque es vendido a Leigh \& Co., Londres, quienes lo usan, al mando del capitán Gardner, al menos hasta $1800^{15}$, como privateer, es decir como buque privado autorizado por el gobierno de Inglaterra para atacar buques extranjeros, especialmente mercantes (Starkey, 1990). Sabemos que el 8 de agosto de 1797 llega a Deal, Inglaterra, el Emelia, Gardner, [procedente] de Jamaica ${ }^{16}$. No sabemos qué ocurre con la nave después de 1800 .

Conocer el nombre real del buque no es tarea sencilla y hemos respetado, hasta ahora, el nombre

cgi/pt?id=hvd $.32044020166658 \& v i e w=1$ up\&seq $=104$ consultado el 26 de abril de 2020.

13 Register of Ships, 1789. En https://babel.hathitrust.org/ cgi/pt?id=hvd $.32044020166310 \& v i e w=1$ up\&seq $=5$, consultado el 31 de marzo de 2020.

14 En https://whalinghistory.org/?s=BS0303, consultado el 31 de marzo de 2020.

15 Register of Ships, 1800. En https://books.google.cl/boo $\mathrm{ks}$ ? id=v6yalbUX6IMC\&pg $=\mathrm{PP} 1 \&$ redir_esc $=\mathrm{y} \#_{\mathrm{V}}=$ onepage $\& \mathrm{q} \& \mathrm{f}=$ false, consultado el 31 de marzo de 2020

16 Lloyd's List (Londres), 11 de agosto de 1797. 
que cada autor le ha dado. No sólo se lo llama Emelia (Webb, 1988; Andress, 2008; Nichols, 2009; Dawson, 2010), sino también Emilia (Stackpole, 1972; Jackson, 1978; Daughan, 2013) o incluso Amelia (Spears, 1908; Melville, 2002 [1851]; Kirk, 2012). En el Lloyd's Register aparece algunos años como Emelia (1782, 1798, 1799), otros como Emilia (1783-1797, 1800), nunca como Amelia. En el Lloyd's List aparece con los tres nombres, aunque domina Emilia. En 1790 el buque es asegurado por dos años, bajo el nombre de Emelia, en $£ 1.800^{17}$.

La razón de su nombre también tiene sus versiones. Según David Alston, la nave fue bautizada con el nombre de Emelia en homenaje a la madre de Evan Baillie, uno de sus propietarios, llamada Emelia Fraser de Reelig (Alston $\mathrm{n} / \mathrm{d})^{18}$, aunque otro autor señala que la madre de Evan Baillie se llamaba, en realidad, Emilia (Hamilton, 2015). Otra versión indica que habría recibido el nombre de Emelia en homenaje a Emelia Vansittart [17381818], viuda de Henry Vansittart, superintendente de la Compañía de las Indias Orientales. La Sra. Vansittart fue una inversionista permanente en el negocio ballenero de los Enderby y muy cercana a los comerciantes londinenses (Dawson, 2010). Esta versión sería satisfactoria si no supiéramos que su nombre es anterior a la adquisición del buque por los Enderby, aunque sí podría ser una razón para no cambiárselo. Lo importante es que el buque fue nombrado de diferentes maneras y esos nombres aparecen en los distintos documentos. En este trabajo ocuparemos, de ahora en adelante, el nombre de Emelia, el primero que aparece en las fuentes (Register of Ships, 1782), sin querer con esto afirmar que ese era el nombre con el que el velero fue siempre conocido.

¿Qué sabemos de James Shields, su capitán durante el periplo ballenero que nos interesa? No demasiado (Jones, 1991). Según la noticia de su muerte, que ocurrió a los 69 años, habría nacido

17 Insurance Ship Emelia (James Shields commander). Collection Royal \& Sun Alliance Insurance Corporation, CLC/B/192/F/001/MS11936/378/58871. London Metropolitan Archives, Londres.

18 Ver http://www.spanglefish.com/slavesandhighlanders/ index.asp?pageid $=670520$, consultado el 30 de marzo de 2020.

19 The York Herald (York), 5 de julio de 1823. en $1754^{19}$. Los textos, en su mayoría, nos dicen que era originario de Nantucket (Spears, 1908; Nichols, 2009; Dawson, 2010), pero no tenemos antecedentes fidedignos que lo prueben.

En 1777, es decir a los 23 años, era enrollador de línea [line-coiler] ${ }^{20}$ en la fragata ballenera inglesa Pitt, de la casa Enderby \& Sons, desde 1778 es arponero en el mismo buque. Ese año, el buque es rebautizado como Sandwich y James Shields continúa, primero como arponero, luego como oficial, hasta que en 1785 asume como su capitán (Jones, 1991). Comanda dos cruceros balleneros a los bancos de Brasil y África, uno entre 1785 y 1786 y otro entre 1786 y 1787 (Jones, 1991). En 1788 se hace cargo del Emelia, fragata que conduce en tres viajes balleneros al océano Pacífico, 1788-1790, 1790-1791 y 17921794. En 1794 asume como capitán del bergantín Laird y lo lleva a un crucero ballenero a las costas de Perú, regresando en 1797. En 1798 asume como capitán de la fragata Mary, que sale en busca de ballenas a las costas de Nueva Holanda (Australia), llegando el 26 de septiembre de 1800 (Jones, 1991).

En 1809, cuando se casa una de sus hijas, ya residía en Norton, Durham, Inglaterra ${ }^{21}$, lugar donde fallece, el 21 de junio de 1823, el primer comandante que fue a la pesca en los mares del sur por el Cabo de Hornos (Richmond, 1868, p. 140).

\section{LOS DOCUMENTOS}

Edouard Stackpole señala que la bitácora del viaje del Emelia no parece haberse salvado, pero asegura que una carta escrita por el capitán James Shields dirigida a Enderby \& Sons, es de dramática importancia para la historia de la caza de ballenas, pues fue redactada desde Deal22, el 6 de marzo de 1790, mientras el buque esperaba marea favorable para poder ascender el Támesis

20 El line-coiler era uno de los tripulantes del bote ballenero que debía asegurar la línea que unía la ballena con el bote, después de ser arponeada.

21 The Hull Packet (Hull), 16 de mayo de 1809.

22 Deal es una localidad de la costa sur inglesa, en el condado de Kent, situada a ocho millas al noroeste de Dover y a ocho millas al sur de Ramsgate. 
y atracar en St. Paul's Wharf, Londres, el último tramo de su regreso a casa (1972, pp. 125-126). Stackpole indica que la carta, que presenta una [inmejorable] imagen gráfica del histórico viaje, se encuentra depositada en la Public Library of New South Wales, Sydney, Australia, como parte de la Colección Chalmers (1972, p. 126). Stackpole utiliza la carta de Shields en forma extensa y minuciosa (1972) y su trabajo es citado por otros investigadores (Francis, 1990; Mawer, 1999).

No se pudo revisar el ejemplar del documento que menciona Stackpole, pero se consultaron tres versiones de la carta. Dos de ellas se encuentran online en el sitio de la National Library of Australia (www.nla.gov.au) ${ }^{23}$. Se trata de microfilmes cuyos originales están, uno en la British Library y el otro en The National Archives, en Londres. El documento de la British Library forma parte de los Liverpool Papers y es definido por la misma biblioteca como una copia ${ }^{24}$. Son dos fojas escritas con letra bastante pequeña, muy apretada, pero bastante legible. El documento de The National Archives es una copia de la carta de Shields, enviada con una nota por Samuel Enderby a Evan Nepean, Subsecretario del Home Office, el 16 de agosto de $1790^{25}$. Se trata de un documento de cuatro fojas, en regular estado de conservación, pero legible. La tercera versión, también una copia, está en la Caird Library \& Archive, National Maritime Museum, Greenwich, Londres, entre los Markham Family Papers ${ }^{26}$. En este caso se trata de un documento de siete fojas, escritas de forma regular, en muy buen estado de conservación y completamente legible (ver Fig. 1). No hay ninguna diferencia en el contenido de las distintas versiones de la carta.

Hay otros documentos relacionados con el viaje que entregan fragmentos de información que permiten tener una idea más precisa de la naturaleza, características, contexto y consecuencias del

23 (1) Letter of Captain J. Shields to Samuel Enderby and Sons, 06 March 1790, https://nla.gov.au/nla.obj761982653/view; (2) Letter of Samuel Enderby to Evan Nepean, enclosing copy of letter from Captain Shields of voyage of Emilia in South Seas (6 March 1790), https:// nla.gov.au/nla.obj-728094411/view. National Library of Australia, Canberra, Australia; consultado el 28 de marzo de 2020.

24 Letter of Captain James Shields, Master of the ship Emilia to S Enderby and Sons, 1790 (copy). Liverpool Papers, periplo ballenero del Emelia en los mares del sur. Algunos de estos papeles no pudimos consultarlos en forma directa sino que usamos referencias de segunda mano, como por ejemplo a Conon Fraser para la carta de Samuel Enderby Jr. a Joseph Banks, del 26 de agosto de 1788 (Fraser, 2014); a William Dakin para una carta de Enderby a George Chalmers, sin fecha, pero seguramente de 1788 (Dakin, 1938); a Edouard Stackpole para la carta del 5 de noviembre de 1789 de los comerciantes londinenses al Consejo de Comercio (Stackpole, 1972); a Joseph R. Slevin para otra carta de Enderby al mismo Chalmers, del 28 de junio de 1790 (Slevin, 1959).

Pero el documento de mayor interés, junto con la carta de Shields es un informe elaborado por los comerciantes londinenses Samuel Enderby \& Sons y Alexander \& Benjamin Champion, enviado el 16 de marzo de 1792 al Committee of Trade \& Foreign Plantation of Great Britain. Este documento es una respuesta escrita frente a un cuestionario sobre la marcha de la Southern Whale Fishery en el océano Pacífico, denominado Head of Enquiry, enviado el 10 de marzo de 1792 por el Committee of Trade \& Foreign Plantation of Great Britain.

Es importante conocer las preguntas del cuestionario, pues refleja el interés del Gobierno en estas materias. El Comité deseaba saber: (1) el tiempo que estuvo fuera de Gran Bretaña cada buque; (2) el nombre de cada puerto en el que recalaron durante todo el viaje y cuánto tiempo permanecieron en cada uno, considerando las fechas de salida de cada puerto y el tiempo demorado en llegar de un puerto a otro; (3) el número de marineros que murieron entre la salida a los mares del sur y su regreso a puerto; (4) la condición de los marineros sobrevivientes; (5) cualquier tipo de dificultad que pudieron haber

Volumen I (Add MS 38225 [Jan 1790-Nov 1790]), fs. 7071. British Library, Londres, Inglaterra.

25 Letter of Samuel Enderby to Evan Nepean, 16 de agosto de 1790. Home Office 42/16, Letters and papers, 1790 Jan 01-1790 to Aug 31, fs. 288-292. National Archives, Kew, Londres.

26 James Shields to Saml. Enderby \& Sons, 6 March 1790. Markham Family Papers, MRK/105/1/113; National Maritime Museum, Caird Library \& Archive, Londres (Greenwich), Inglaterra. 


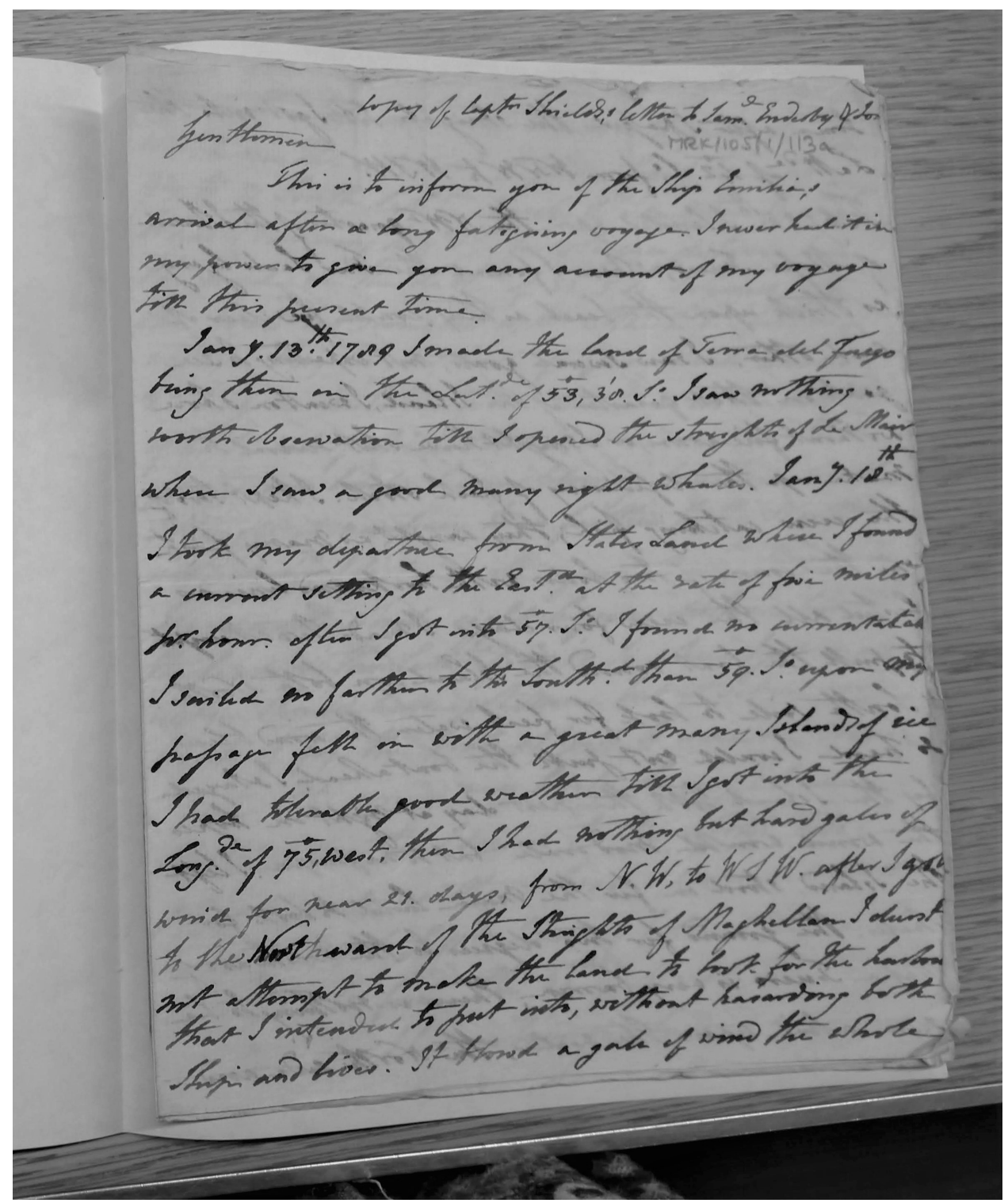

Fig. 1. Fotografía de la primera foja de la carta de James Shields a Enderby \& Sons, 1790 [MRK/105/1/113; National Maritime Museum, Caird Library \& Archive, Londres (Greenwich), Inglaterra].

tenido en llevar el barco, sea alrededor del cabo de Hornos, sea a los puertos que tocaron o a Casa, y las medidas que tuvieron que tomar para obviar estas dificultades; (6) en qué latitud y a qué distancia de la costa desarrollaron sus pesquerías; (7) el estado de cualquier puerto en cualquier isla en los mares del sur o sobre la costa española en el

27 Question to be submitted to Enderby \& Champion on Southern Whale Fishery, March $10^{\text {th }}$, 1792. Liverpool oeste de Sudamérica, donde los buques empleados en la Southern Whale Fishery podrian dirigirse por provisiones y bajo qué circunstancias no serían buenos puertos de comercio ${ }^{27}$.

En el informe se transcribe un par de párrafos de la muchas veces copiada carta de James Shields y también se hace referencia al diario del viaje, 
indicando que los cachalotes "se habrían cazado entre los paralelos $8^{\circ}$ y $32^{\circ}$ latitud sur" 28 . Pero la importancia del escrito es que no sólo informa sobre las características del crucero del Emelia, sino que también ofrece una serie de datos relevantes sobre cada uno de los siete viajes de balleneros ingleses que fueron al océano Pacífico por la ruta del Cabo de Hornos, siguiendo el ejemplo del Emelia. Todos estos viajes se iniciaron en 1790, después de la llegada de Shields a comienzos de marzo de 1790.

Las respuestas de los comerciantes londinenses son leídas en la sesión del 18 de marzo de 1792 del mencionado Comité y discutidas por sus miembros ${ }^{29}$.

\section{EL RELATO OFICIAL}

Samuel Enderby Jr. le envía una carta a George Chalmers, secretario de Lord Hawkesbury, Presidente del Consejo de Comercio del Reino Unido, sin fecha, donde le informa de la compra y apresto de un buque que enviará a la caza de cachalotes en los mares del sur, cruzando el cabo de Hornos:

Hemos comprado y adaptado un fino buque con grandes gastos para ir a rodear el Cabo de Hornos; está listo para zarpar, somos los únicos propietarios que intentan enviar un buque a esta rama de las pesquerías; parece ser la opinión general de la mayoría de las personas que conoce aquellos mares que las ballenas de espermaceti están al norte de nuestros limites. Como parecemos ser los únicos aventureros que desean arriesgar su propiedad a tan gran distancia para la exploración de una pesquería, humildemente solicitamos a sus señorías (si la petición no es impropia) que en el caso

28 Particulars relating to Every English Vessel which has gone into and returned from the Whale Fishery in the Pacific Ocean, 1792. Records on the Board and Trade, BT 1/2 part 2, (October 1791-March 1792) fs. 271-274. National Archives, Kew, Inglaterra. En adelante lo citaremos como Enderby, Samuel \& Alexander Champion, 1792.

29 Reply by whaling merchants to questions about Southern Whale Fishery. Liverpool Papers, Volumen I (Add MS 38393, [6 Jan 1791-31 Mar 1792]), fs. 205-206. British de no encontrar ballenas de espermaceti al sur de la linea en el Océano Pacífico Sur nuestro buque o cualquier otro velero empleado en la pesquería, tenga permiso para navegar hasta los 24 grados de latitud norte, pues se sabe que hay ballenas de espermaceti en la costa española, aunque no es seguro si se encuentran al sur o al norte de la línea. Del éxito de nuestro buque depende el establecimiento de la Pesquería en el Océano Pacífico Sur, pues muchos propietarios han declarado que esperarán hasta escuchar si nuestro buque es exitoso; si lo es una amplia rama de la pesquería se desarrollará en aquellos mares; si no lo es, nosotros pagaremos por el conocimiento (Dakin, 1938, p. xxvii) ${ }^{30}$.

También le escribe el 26 de agosto de 1788 a Joseph Banks diciéndole que tiene un buen barco, el Emilia, listo para partir en una semana a la pesca de ballenas del sur alrededor del Cabo de Hornos y le pregunta si Juan Fernández está poblado y si es así, si habría algún riesgo para su barco de ser incautado si recalaba en el lugar; si sabe si hay cachalotes alrededor del Cabo; si hay algunos buenos mapas de ahí y le ofrece si necesita algo que pueda procurarle, en cambio (Fraser, 2014, p. 41)

Para los comerciantes londinenses el apoyo de las autoridades gubernamentales era fundamental. En una carta escrita el 5 de noviembre de 1789 al Consejo de Comercio plantean lo siguiente:

No somos competentes para indicar cuan cerca de la costa española, al oeste del Cabo de Hornos, será necesario llevar nuestra caza de cachalotes para ser servida con éxito, pero de toda la información que podemos disponer suponemos que será cerca de la

Library, Londres, Inglaterra.

30 El documento no tiene fecha; aunque Dakin estima que es de comienzos de 1789 , creemos que más bien es de 1788 , pues en 1789 el buque estaba cerca del cabo de Hornos.

31 Fraser señala que la carta original se encuentra en la Library and Archives, Royal Botanic Garden, Kew, Londres, JBK/1/4 Joseph Banks Correspondencia, volumen 1, folio 319, c 1780 (Fraser, 2014, p. 221). 
costa. Pensamos que si tenemos la libertad pescar a 5 leguas de la costa sería tan cerca como deseamos. Muchos cachalotes han sido vistos alrededor de la isla de Juan Fernández. Suponemos que no habrá objeciones de hacerlo cerca de cualquiera de las islas, siempre y cuando no se trate de comerciar. Hay una isla cerca de Juan Fernández en el Mar del Sur llamada Más Afuera, en la que hay muchos lobos. ¿Si no está habitada, se puede enviar botes a matar los lobos? (Stackpole, 1972, p. $123)^{32}$.

La doctrina de las cinco leguas será considerada en los acuerdos que firmen los ingleses con los españoles en la Convención de Nootka de 1790.

En la sesión del 9 de marzo de 1790 del Council Chamber of Whitehall, se lee una carta de Samuel Enderby Jr. donde informa del arribo a salvo a Gravesend de un buque empleado en la Southern Whale Fishery, que había llegado al Mar del Sur por el Cabo de Hornos y que era el primer y único buque que ha seguido dicha trayectoria en esta pesquería ${ }^{33}$. Enderby le reitera a Chalmers, en una carta escrita el 28 de junio de 1790, que la fragata Emilia, capitán James Shields, regresó en marzo pasado de un viaje ballenero a las costas de Perú, y que como este buque [...] fue el primero en cazar ballenas en el Océano Pacífico Meridional, pusimos a bordo una cantidad extra de todas nuestras mercancías, para preservar mejor la salud de la tripulación en tan inhospitalaria costa (Slevin, 1959, p. 52) ${ }^{34}$. Esta información queda registrada nuevamente en el informe que elabora el 3 de julio de 1790 James Cottrell, de la Oficina del Tesoro, relacionada con el hallazgo

32 Stackpole indica que la carta se encuentra en Records of the Board of Trade BT 6/95, f. 187. National Archives, Kew, Inglaterra.

33 Letter from Samuel Enderby on first whaling vessel to round Cape Horn into South Sea. Serie Papers of Charles Jenkinson, $1^{\text {st }}$ Earl of Liverpool, Subseries Add. MS 3838838394, ítem f. 71. British Library, Londres, Inglaterra.

34 Slevin, desafortunadamente, no indica el archivo específico donde se encuentra esta carta ni las demás que cita.

35 Council recommending a stay of process against some spirits belonging to Messrs. Enderby \& Sons returned in the ship Emilie from the Southern Whale Fishery. Public de licores a bordo del Emelia a su llegada del viaje al cabo de Hornos ${ }^{35}$.

El viaje del Emelia no pasa desapercibido y su buena fortuna es difundida posteriormente a través de diversos periódicos en todo el Reino Unido:

El buque Emilia, propiedad del Sr. Enderby, es el único ballenero del sur que ha rodeado el Cabo de Hornos y ha hecho el viaje más exitoso de un buque de ese comercio. De su ejemplo, muchos otros, sin duda, se verán inducidos a comprometerse en el mismo emprendimiento $y$, si son exitosos, no sólo la importación de aceite de Norteamérica se hará innecesaria sino también disminuirá la demanda de sebo de nuestros buenos amigos rusos ${ }^{36}$.

\section{EL VIAJE DEL EMELIA}

Tenemos distintas fechas para el zarpe del buque desde Londres. Edouard Stackpole habla, con mucha propiedad, de la "mañana de agosto de 1788, cuando las amarras del Emilia fueron soltadas en el Paul's Wharf y el buque, con la marea, baja rápidamente el Támesis" (1972, p. 125). Bill Spence asegura que "sale de Londres el 7 de agosto de 1788” (1980, p. 63). Jane Clayton indica que el buque zarpa en el mes de septiembre de 1788 (2014). Robert L. Webb (1988, p. 12) dice que lo hace el $1^{\circ}$ de septiembre de $1788^{37}$. La información que entrega la literatura ballenera no es muy precisa. La fecha más probable es aquella que fija la salida de Paul's Wharf, el muelle de los Enderby en el Támesis, el 11 de septiembre de $1788^{38}$.

Record Office, Treasury, T1/682/1369, fs. 293-295. National Archives, Kew, Inglaterra.

36 Hampshire Chronicle (Hampshire), 20 de noviembre de 1790; Caledonian Mercury (Edimburgo), 27 de noviembre de 1790; Hereford Journal (Hereford), 1 de diciembre de 1790 .

37 El Emelia zarpa de Londres el 7 de agosto de 1788, regresando el 9 de marzo de 1790, luego de 583 días de navegación, con una cosecha de 140 toneladas de aceite de esperma (https://whalinghistory.org/?s=BV030300; consultado el 28 de marzo de 2020.

38 Caledonian Mercury (Edimburgo), 21 de agosto de 1790. 
El 29 de septiembre está anclado en Downs, esperando zarpar en su viaje a los mares del sur ${ }^{39}$ (en la Fig. 2 se muestra el itinerario de ida del Emelia en su viaje al océano Pacífico). La nave llega a Falmouth, en el sur de Inglaterra, y Samuel Enderby Jr. informa que zarpa de este puerto recién el 11 de octubre de 1788, rumbo a Las Palmas, en la Gran Canaria. Sale de Las Palmas el 31 de octubre, después de haber permanecido dos días en el puerto, y llega a las islas de Cabo Verde el 11 de noviembre de 1788; zarpa el 20 del mismo mes y se dirige a Río de Janeiro ${ }^{40}$, donde, luego de reparar una pequeña fuga en el casco, sale el 25 de diciembre rumbo al cabo de Hornos ${ }^{41}$.

Desde este punto, podemos seguir el derrotero narrado por el propio Shields en su carta a Enderby \& Sons ${ }^{42}$ : el 13 de enero de 1789 llega a las costas de Tierra del Fuego por el estrecho de Le Maire donde pudo ver una gran cantidad de ballenas francas, pero no trató de cazarlas. El 18 de enero sale de la isla de los Estados rumbo al cabo de Hornos; las corrientes lo llevan hasta los $57^{\circ}$ $\mathrm{S}$ donde se encuentra con muchas islas de hielo. Luego de luchar con poderosos vientos el Emelia logra enderezar hacia el norte, pasa por la boca occidental del estrecho de Magallanes y en latitud $42^{\circ} \mathrm{S}$ vira hacia el NW. El 23 de febrero de 1789 llega a la isla de Más Afuera, en el archipiélago de Juan Fernández.

El primer punto de tierra que tocan los balleneros en el Pacífico es la parte noroccidental de la isla de Más Afuera, donde encontramos lobos marinos que yacian tan densos en la playa como les permitía el espacio libre que había entre ellos; vimos varias cabras, las que no esperábamos encontrar en este sector de la isla; no bajé con armas de fuego a tierra o podría haberle disparado a varias para compensar la deficiencia de provisiones frescas. Zarpan rumbo noreste y el 3 de marzo de 1789, cuando estaban en latitud $31,2^{\circ}$ sur (un poco al norte de

39 Lloyd's List (Londres), 30 de septiembre de 1788

40 Enderby, Samuel \& Alexander Champion, 1792.

41 Lloyd's List (Londres), 27 de marzo de 1789.

42 James Shields to Saml. Enderby \& Sons, 6 March 1790 (copy, 2 pages). Markham Family Papers, MRK/105/1/113; National Maritime Museum, Caird Library \& Archive, Londres (Greenwich), Inglaterra. La citaremos como Carta de Shields a Enderby, 1790. la altura de Tongoy), y a 25 leguas de tierra firme (unos $140 \mathrm{~km}$ ), capturan sus primeros cachalotes. Shields habla de cinco ejemplares muertos y cuatro escapados. Continúa hacia el norte, obteniendo, hasta el 7 de mayo, 105 toneladas $^{43}$ de aceite de esperma entre los $32^{\circ}$ y $17^{\circ}$ latitud sur. En ese momento Shields decide ir a cazar lobos, pero como no era temporada en la isla de Más Afuera, se dirige a la isla de los Lobos, a $7^{\circ}$ latitud sur y a doce leguas de la costa, donde desembarca el 17 de mayo. Obtiene 870 pieles de lobo fino. El 2 de junio zarpa de la isla rumbo al sur, para continuar con la persecución de cachalotes. Las corrientes lo llevan al suroeste y en la latitud $23^{\circ}$ sur estaba a 300 leguas de tierra firme. Sin embargo, puede volver y el 5 de julio toca tierra en $16,20^{\circ}$ latitud sur (a la altura de Atico, al norte de Mollendo, Perú), encontrando el lugar despoblado, pero "pleno de cachalotes". La cantidad de aceite a bordo llega a las 147,5 toneladas y el capitán decide regresar el 15 de agosto de 1789 a casa, sobre todo por los estragos que hace el escorbuto desde hace un mes en la tripulación ${ }^{44}$.

El regreso sigue la misma ruta. El 5 de septiembre pasa por la isla de Más Afuera y el 23 de septiembre cruza el cabo de Hornos. El 17 de octubre recala en Río de Janeiro ${ }^{45}$. Permanece casi dos meses en este puerto y el 10 de diciembre de 1789 sale rumbo a Inglaterra. Llega a Dover el 6 de marzo de $1790^{46}$, y el 12 de marzo entra finalmente en Gravesend ${ }^{47}$. El capitán señala que desde que salió de las costas de Brasil no vio ningún otro buque ballenero ${ }^{48}$. Se expresa de manera muy satisfactoria del Emelia: El buque navega notablemente rápido y es uno de los mejores en los que he tenido los pies a bordo en una fuerte tormenta de viento; no tensa los aparejos y es uno de los barcos más completos para esta pesca de ballenas alguna vez construido ${ }^{49}$.

La productividad del viaje es notable. Shields señala que cazamos 27 ballenas, de las que 21 eran

43 Corresponde a la tonelada imperial inglesa, equivalente a 955 litros o 0,995 toneladas métricas (Zupko, 1985).

44 Carta de Shields a Enderby, 1790.

45 Carta de Shields a Enderby, 1790.

46 Enderby, Samuel \& Alexander Champion, 1792.

47 Lloyd's List (Londres), 16 de marzo de 1790.

48 Carta de Shields a Enderby, 1790.

49 Carta de Shields a Enderby, 1790. 


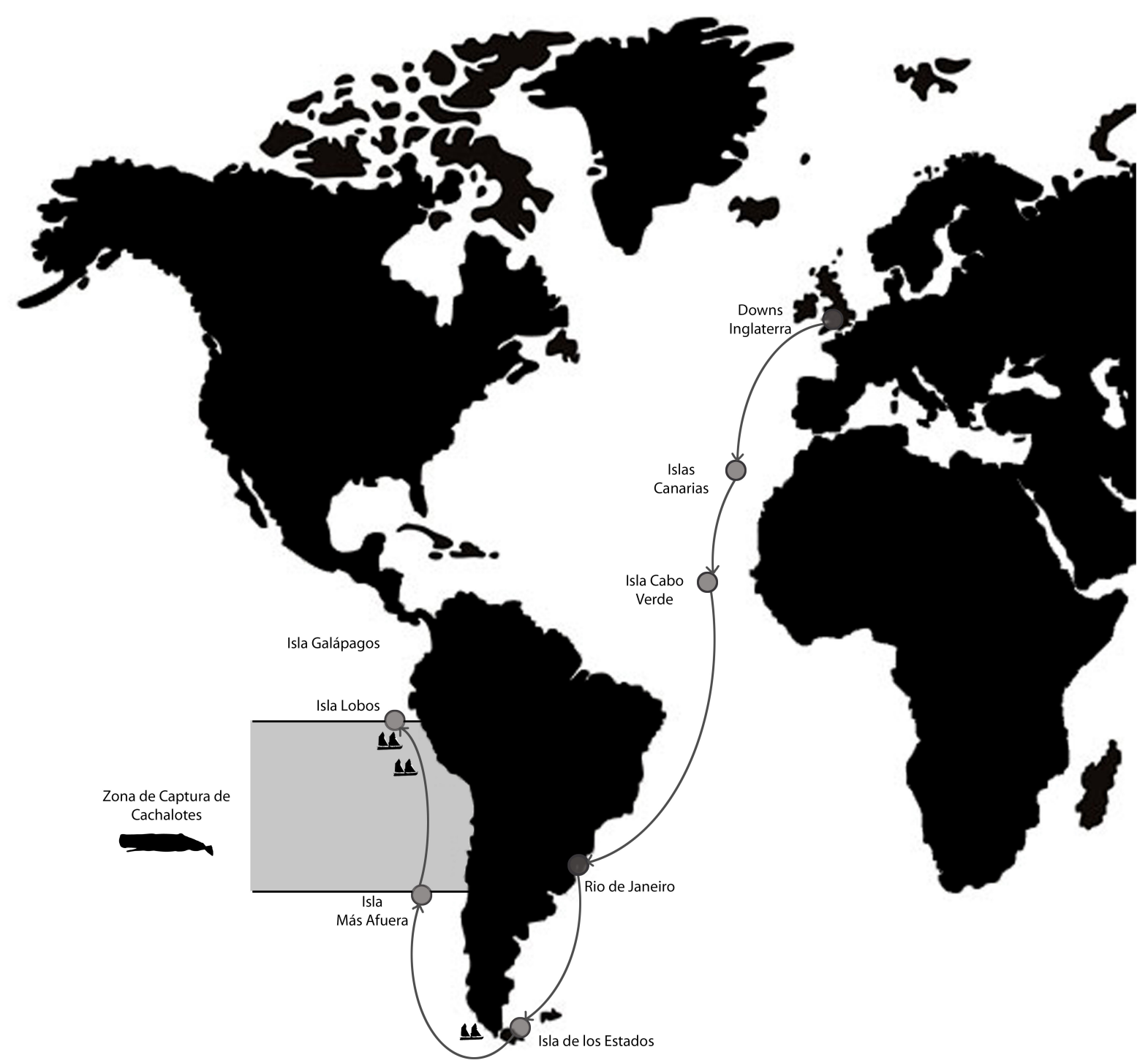

1. Encuentros con buques españoles

Cabo de Hornos

Viaje de Emelia (1788-1790)

Fig. 2. Representación del viaje de ida del Emelia [1788-1789], capitán James Shields, al océano Pacífico [dibujo de Daniela Quiroz].

grandes y a pesar de haber perdido doce barriles debido a un mal almacenaje en la bodega, espero que el aceite rondará entre las 143 y 144 toneladas de las cuáles 41 corresponden a espermaceti. En el viaje se obtuvieron también 870 pieles de lobo ${ }^{50}$.

Las expediciones balleneras eran, en general, de amplio espectro. Buscaban ballenas de barbas, cachalotes, lobos comunes, lobos finos, incluso nutrias. La carne no era una mercancía valiosa por los empresarios balleneros de la época pues no tenían a bordo la tecnología necesaria para transformarla en aceite. Eso sólo ocurrirá en el siglo XX con la caza moderna de ballenas. En el viaje del Emelia se buscaban cachalotes y 
lobos finos. Es notable que hayan visto ballenas francas en el estrecho de Le Maire y no trataran de cazarlas.

Las estimaciones usuales señalan que de un cachalote de tamaño mediano se puede obtener, en promedio, ocho barriles de espermaceti y unos 30 a 40 barriles de aceite del resto del cuerpo (Ellis, 2011). En este crucero se capturaron 27 ejemplares que produjeron 143.5 toneladas [145 toneladas si tomamos en cuenta los 12 barriles perdidos], de las cuales 41 toneladas eran de espermaceti [head matter]. Si consideramos que una tonelada es igual a ocho barriles (Zupko, 1985), tenemos que la productividad de espermaceti fue de 328 barriles y la del resto del aceite [sperm oill fue de 832 barriles [con los barriles perdidos]. Como los cachalotes capturados fueron 27 , se obtuvo en el viaje un promedio por cada ejemplar procesado de 12 barriles de espermaceti y 31 barriles de aceite. El espermaceti representa más de un tercio del volumen de aceite obtenido, muy por encima del promedio histórico, que era un quinto del total de aceite de esperma ${ }^{51}$.

En términos económicos el viaje del Emelia le produce a Enderby \& Sons la increíble cifra de $£$ $11.485^{52}, £ 1.745 .720$ en moneda actual.

\section{CONTACTOS CON BUQUES ESPAÑOLES}

El capitán Shields relata en su carta que el Emelia tiene dos encuentros con mercantes españoles. El primero sucede el 9 de junio de 1789 en $8^{\circ}$ latitud sur cuando se topa con un buque que iba de Guayaquil a Lima, a cuyo capitán le pidió que llevara un par de cartas, pero éste se niega, dudando que vinieran a cazar ballenas desde Londres. El segundo acontece el 5 de julio en $16^{\circ}$ latitud sur con una embarcación que iba de Chile a Lima, cuyo capitán fue "más amable", intercambiando mercancías por algunas provisiones frescas. Estos dos encuentros se ajustan con la declaración efectuada el 17 de enero de 1791 por Samuel

51 An account of Number of Ships with their Cargoes, which returns from the Southern Whale Fishery to England in the year 1788, as furnished by Mr. Enderby, one of the Adventurers. Public Record Office, PRO 30/8/259, f. 160. National Archives, Kew, Londres.

52 Para este cálculo usamos la siguiente fórmula: $\mathrm{P}$ $($ productividad $)=(41$ toneladas de espermaceti*£68+
Enderby Jr. ante el Committee of Trade \& Foreign Plantation, indicando que cuando el Emilia fue en su viaje de pesca al oeste del cabo de Hornos llegó hasta la latitud 7 Sur y no se encontró con ningún buque de guerra español sino sólo con dos mercantes, que se alarmaron mucho, y uno de ellos les proporcionó algunas provisiones frescas $^{53}$.

Es un desafío encontrar fuentes hispanas que registren el paso del Emelia por las costas sudamericanas pues el buque no toca ningún punto poblado en su viaje. Contamos afortunadamente con una referencia del historiador español Felipe Valdés Hansen (2014) y con los antecedentes que entrega el historiador peruano Jorge Ortiz Sotelo (2015). La revisión de estos trabajos sugiere que la fragata inglesa tuvo contacto con tres buques mercantes españoles y no dos como lo indica el capitán Shields.

Ortiz Sotelo (2015) señala que el Emelia se encuentra con buques mercantes españoles dos veces, utilizando como fuente de información las Memorias de Gobierno del Virrey Teodoro de Croix (1859 [1790]). El primero habría ocurrido en la altura de 50 grados al sur cuando la fragata Nuestra Señora de Los Placeres, capitán Pedro Pineda, de la Compañía de Filipinas, se topa con una fragata inglesa forrada en cobre, cuyo capitán había pasado a bordo de Los Placeres y le dijo a ésta que venía a la pesca de ballenas (de Croix, 1859, p. 259; cf. Ortiz Sotelo, 2015, p. 166). El otro sucede el 25 de abril de 1789, cerca del puerto de Ilo, cuando un navío inglés, que acaso sería el mismo que también se había avistado cercano a la caleta de Mollendo, intercepta al barco nombrado La Tartana, que iba a cargar huano a la isla de Patillo, informando el capitán, de nacionalidad portuguesa, que habian pasado en un bote cinco ingleses a La Tartana con las demostraciones de paz que significaban por las voces 'no pelea, no pelea' que articularon: que se informaron del piloto de La Tartana de

143,5 toneladas de aceite de esperma* $£ 60+870$ pieles de lobo*£0,1)

53 [Declaración de Samuel Enderby ante el Committee of Trade \& Foreign Plantation, 17 de enero de 1791]. Serie Papers of Charles Jenkinson, $1^{\text {st }}$ Earl of Liverpool, Subseries Add. MS 38393, ff. 10-11 British Library, Londres, Inglaterra. 
los puertos y caletas de aquella costa y de los lugares donde había ballena: que habiéndose hecho reciprocamente unos pequeños obsequios, se habian vuelto a bordo de su fragata, que al parecer estaba recién ensebada y pintada de amarillo: que habian dicho haber salido de Londres y haber arribado al Geneiro: y que así, a proa, como en la toldeta de popa, conducian varios botecitos propios para la operación de la pesca de ballenas: que separada La Tartana de la fragata, que sería como de 24 a 26 varas de quilla, se había enmarado, hasta perderse de vista; y La Tartana, no teniendo viento favorable para regresar a Ilo, siguió su viaje a Patillo (de Croix, 1859, p. 260; cf. Ortiz Sotelo, 2015, pp. 166-167). Teodoro de Croix asume que la embarcación avistada y que abordó a La Tartana parecía ser la misma que encontró la fragata Placeres y que advirtió venir proveída de utensilios para la pesca de la ballena (de Croix, 1859, p. 261).

Valdés Hansen habla de un tercer encuentro, ocurrido el 5 de julio de 1789, entre el pailebot español La Rosa y una fragata inglesa, que cree es la Emilia. El capitán del pailebot informa que fue abordado a la altura de Morro Quemado y de la isla de los Lobos [...] por una fragata inglesa, mientras cubría la última etapa de su travesía entre Chiloé y El Callao; se identificaron como balleneros que habían capturado diez y nueve ballenas. Valdés Hansen agrega que eran quizás los mismos que más al sur, en abril, habian abordado otro barco con igual modus operandi: al anochecer para aprovecharse de la oscuridad, subiendo a bordo sin previo aviso y calculada intimidación para llevarse o intercambiar aquello que necesitaban $(2014 \text {, p. 44) })^{54}$.

La carta del capitán Shields no informa sobre el encuentro ocurrido en los $50^{\circ}$ sur, solamente de los dos posteriores, donde coinciden los lugares, pero no las fechas. El encuentro cerca de la isla de los Lobos sucede, según las fuentes españolas, el 5 de julio y no el 9 de junio como lo manifiesta

54 Valdés Hansen señala que el documento original se encuentra en Archivo General de Indias, Chile, legajo 211, $n^{\circ} 88$, fs. 764-767. Se trata de una carta de Ambrosio O'Higgins, presidente de la Audiencia de Chile, escrita en Santiago el 8 de octubre de 1789 y dirigida a Antonio Valdés, secretario de Hacienda de Indias, que incluye un
Shields, quien afirma que el encuentro del 5 de julio habría sido cerca del puerto de Ilo (Perú), el que, según las fuentes hispanas, habría ocurrido meses antes, el 25 de abril. Estas discordancias no son tan extrañas sobre todo sabiendo que la carta de Shields fue escrita bastantes meses después de ocurridos los hechos.

Uno se podría preguntar por qué España, que tenía un dominio completo del océano Pacífico, el "lago español" (Spate, 1979) como se lo conocía, no desarrolló alguna actividad ballenera en sus aguas antes de la llegada de los ingleses. Los españoles no contaban con la capacidad técnica necesaria para desarrollar una actividad ballenera exitosa. Su único intento fue la creación de la Real Compañía Marítima, con operaciones balleneras en el Atlántico Sur entre 1790 y 1805, tan dificultadas por la carencia de personal especializado que se tuvo que contratar arponeros y otros especialistas en Inglaterra y Estados Unidos. Se aprobaron algunos proyectos a fines del siglo XVIII para cazar ballenas en el Pacífico Oriental, pero ninguno alcanzó a ejecutarse (Quiroz, 2020).

\section{ARCHAELUS HAMMOND, EL MITO}

Stackpole nos cuenta que, en el antiguo cementerio de Nantucket, conocido como Old North, está la tumba del capitán Archaelus Hammond, el primer hombre en capturar y matar un cachalote en aguas del Pacífico (1972, p. 145). Esta afirmación es recogida y repetida posteriormente en innumerables ocasiones, sin mayores cuestionamientos (Webb, 1988; Dolin, 2007; Nichols, 2009).

Tenemos algunos datos biográficos de Archaelus Hammond. Nace en Nantucket el 29 de mayo de 1759, hijo de Amaziah y Lidia Gardner. Se casa con Priscilla Gardner, hija de John y Mary Coffin. Fallece el 2 de julio de 1830, con 71 años de edad ${ }^{55}$.

En 1784, con 25 años, es teniente en la fragata francesa Compte de Vergennes, capitán

oficio del 20 de septiembre de 1789 del gobernador de Valparaíso relatando las noticias transmitidas por el capitán del paquebote La Rosa.

55 Ver en http://www.lynnesgenealogy.com/ HammondDescendants/website/b3166.htm\#P3171, consultado el 31 de marzo de 2020. 
Richard Coffin, en un viaje ballenero a las costas de Brasil, que se inicia el 11 de octubre de ese año y termina el 25 de enero de 1787. El 14 de octubre de 1787, lo tenemos como teniente del bergantín ballenero francés Renomme, capitán Aaron Sheffield, en un viaje de Dunquerque a las islas de Cabo Verde, que finaliza el 25 de junio de 1788. Entre septiembre de 1788 y marzo de 1790 será el primer oficial (teniente) de la fragata ballenera británica Emelia. El 28 de mayo de 1792 asume como capitán de la fragata ballenera francesa Penélope, en un viaje a los bancos de Brasil, regresando el 22 de abril de 1793. No sabemos qué pasa con el capitán Hammond entre 1793 y 1802. El 3 de agosto de 1802 es capitán de la fragata ballenera francesa $C_{y r u s}{ }^{56}$ que sale de Dunquerque para cazar ballenas en aguas sudafricanas, siendo capturado el 25 de septiembre de 1803 en Delagoa Bay, en la costa oriental de África, por el privateer británico Scorpion. Archaelus Hammond regresa a Nantucket, donde fallece en 1830.

¿En qué momento y bajo qué circunstancias surge la "noticia" en la literatura ballenera? Las historias más antiguas, como la de Thomas Beale (1839) no la recoge; tampoco lo hace Obed Macy (1835), a pesar de que su obra está centrada en la isla de Nantucket, la patria de Hammond. Por lo tanto, creemos que la emergencia de este relato es bastante más tardía.

Charles Scammon indica, en 1874, que el primer ballenero en aventurarse en el Pacífico fue enviado desde Inglaterra en 1787 por la colonia de balleneros de Nantucket y el primer oficial del buque, Archetus Hammond mató el primer cachalote conocido que ha sido capturado en ese océano (1874, p. 209). Scammon cita como su referencia la información que aparece en uno de los números de los Proceedings of American Antiquarian Society. Son las actas de la reunión de la sociedad efectuada el 21 de octubre de 1871 , publicadas en 1872 , donde se incluye, como pie de página, un memorándum enviado por Frederick C. Sanford, conocido historiador de Nantucket, que dice lo siguiente: El primer ballenero que entró

56 Cyrus, fragata de 305 toneladas, construida en Salem, Beverly, para William Leach, Beverly, Isaac Thorndike, Beverly, y Thomas Dickerson, Boston. Capitán, William Leach. Registrada el 7 de julio de 1800 (Essex Institute 1906). al Pacífico lo hizo desde Inglaterra en 1787. El buque fue enviado por la colonia de balleneros de Nantucket en Inglaterra. El capitán Archetus Hammond fue primer oficial de ese barco y mató el primer cachalote que se sabe fue tomado en dicho océano (Sanford, 1872, pp. 28-29) 57. Según nuestras búsquedas, es la primera vez que se menciona este suceso en forma escrita.

Diez años más tarde, Sanford (1884, pp. 216-217) reitera esta información, agregando nuevos datos y modificando los entregados previamente:

En 1778 Mr. Enderby envió a Brazil Banks, la fragata Aurelian ${ }^{58}$, comandada por James Shields, que tenía como su primer oficial a Archelus Hammond. Debido a que llegaron demasiado tarde para la temporada, Mr. Hammond indujo a su capitán a dirigirse al Pacífico, rodeando el cabo de Hornos, que alcanzaron a su debido tiempo y donde comenzaron a cazar ballenas. Mr. Hammond, poco después, capturó y mató al primer cachalote. El buque pronto fue llenado con aceite y se dirigió a El Callao, Perú, para reclutar gente, $y$ de ahí salió para Londres, hablando en el viaje a casa, cerca de Trinidad, $20^{\circ}$ latitud, con la fragata Hope, capitán Thaddeus Swain, cuarenta y seis dias de Dunquerque, rumbo a Delago Bay, llegando en septiembre de 1790.

El relato es repetido por Alexander Starbuck en su notable historia de la industria ballenera estadounidense, escrita en 1877 y publicada en 1878, curiosamente también como nota a pie de página: en el Pacífico, los estadounidenses han sido precedidos por el Amelia, capitán Shields, un buque armado en Inglaterra, pero manejado por la colonia de balleneros de Nantucket, que navegó hacia ese océano en 1787 y su primer oficial Archelus Hammond mató el primer cachalote conocido que ha sido tomado en dicho

57 Este memorándum fue también publicado en el periódico The Friend (Honolulu), $1^{\circ}$ de mayo de 1872.

58 Primera y única vez que aparece con este nombre el buque Emelia, un error del que desconocemos su causa. 
océano (Starbuck, 1878, p. 90). Esta afirmación es luego tomada por otros historiadores de fines del siglo XIX (cf. Clark, 1887).

Podemos situar entonces el origen de este relato en los escritos de Frederick C. Sanford (1802-1890), no sólo un historiador de Nantucket sino también un importante comerciante, armador de buques y ballenero de Nantucket. Nathaniel Philbrick lo etiqueta como "the mythmaker", un hacedor de mitos. En 1856, luego de su retiro, Sanford inicia lo que sería su proyecto de 35 años: la redención de Nantucket desde un presente casi estático, mediante la evocación de un pasado incesantemente heroico, casi glorioso (Philbrick, 2011, p. 238). Philbrick señala que cuando habia que agregarle gloria a su isla natal, Sanford estaba perfectamente dispuesto a ser flexible con los datos (2011, p. 247). Su mirada sobre la travesía del Emilia, una empresa organizada por "la colonia de balleneros de Nantucket en Inglaterra", donde Archaelus Hammond, de Nantucket, no solamente "indujo a su capitán a dirigirse al Pacífico" sino también "mata al primer cachalote que se sabe fue tomado en dicho océano", parecen ser un ejemplo de estas "flexibilidades" del historiador con los datos, considerando "un bien mayor": la gloria de Nantucket y sus habitantes.

¿Hammond hizo realmente lo que se dice que hizo? No hay fuentes primarias que lo confirmen. Lawrence Briggs afirma que Sanford decía que había conocido bien a Hammond (Briggs, 1889, p. 167). Stackpole, por su parte, señala que Sanford habría escuchado la historia de otros balleneros que conocieron el suceso por el mismo Hammond (1972, p. 127). Finalmente, Stackpole y Summerfield (1974, p. 106) indican que la hazaña [de Hammond] quedó debidamente registrada en una carta escrita por uno de los Enderby, los propietarios del buque, pero no dan señales que permitan ubicar dicha carta.

\section{SOBRE LOS PASOS DEL EMELIA}

Como ya lo habíamos mencionado, la llegada del Emelia a Londres en el mes de

59 Enderby, Samuel \& Alexander Champion, 1792.

60 Lloyd's List (Londres), 19 de marzo de 1790.

61 Lloyd's List (Londres), 19 de marzo de 1790.

62 Lloyd's List (Londres), 23 de marzo de 1790.

63 Register of Ships, 1786. En https://babel.hathitrust.org/ marzo de 1790 provoca la salida de varios buques en busca de cachalotes cruzando el cabo de Hornos. El documento de Enderby \& Sons y Champion Bros. del 16 de marzo de $1792^{59}$ ofrece información importante sobre los viajes de siete balleneros ingleses, todos iniciados en 1790: se trata de las expediciones de las fragatas Greenwich, capitán Locke (1790-1791), Atlantic, capitán Basset (1790-1791), Emelia, capitán Shields (1790-1791) y Kent, capitán Pease (1790-1792), todos de Enderby \& Sons, Londres; fragata New Hope, capitán Dagget (1790-1791), de Thomas Yorke, Londres; fragata Bellisarius, capitán Anderson (1790-1791), de James Mather, Londres; fragata Aurora, capitán Butler (1790-1792), de Sydenham Teast, Bristol. Si se le agrega un octavo viaje, el de la fragata Venus, capitán Coffin (1790-1792), de Champion \& Co., Londres, se completa la lista de los "ocho buques, cuatro de ellos de Enderby \& Sons, que se aventuraron en el Pacífico" en 1790 para cazar cachalotes (Jackson, 1978, p. 109) y regresaron entre 1791 y 1792. Las principales dificultades de este tipo de viaje tienen que ver, según los propietarios de los buques, con las particulares características del clima de la zona del cabo de Hornos y la aparición del escorbuto en viajes largos, sin poder recalar en puertos donde aprovisionarse de productos frescos. No es la presa el problema.

El primer buque en salir, pocos días después del arribo de la Emelia, fue la fragata Greenwich, al mando del capitán John Locke, que zarpa de Downs el 17 de marzo de $1790^{60}$ llegando al día siguiente a Portsmouth ${ }^{61}$. Sale de este puerto el 21 de marzo, rumbo a los mares del sur ${ }^{62}$.

El Greenwich era una fragata construida en 1770 en Liverpool (Clayton, 2014). S. Enderby \& Sons adquiere el buque en 1786 y lo registra en Londres, con 270 toneladas, destinándola a la Southern Whale Fishery ${ }^{63}$. Entre 1786 y 1790 realiza tres viajes a Brazil Banks, todos con John Locke como su capitán: entre 1786 y 1787 obtiene 10 ton $^{64}$ de aceite de esperma, 94 ton de

cgi/pt?id=hvd $.32044020166658 \&$ view $=1$ up\&seq $=147$ consultado el 27 de abril de 2020 .

641 ton o 1 tun equivale a 210 galones o 955 litros en la nomenclatura imperial inglesa (Zupko, 1985). 
aceite de ballena, $71 \mathrm{cwt}^{65}$ (quintales) de barbas de ballena; entre 1787 y 1788 produce 11 ton de aceite de esperma, 125 ton de aceite de ballena, 109 quintales de barbas de ballena y 4.723 pieles de lobo; finalmente, entre 1788 y 1790 alcanza 11,5 ton de aceite de esperma, 92 ton de aceite de ballena, 71 quintales de barbas de ballena y 350 pieles de lobo ${ }^{66}$.

El 12 de abril de 1790 llega a Tenerife, islas Canarias, y el 21 de abril a St. Jago, en las islas de Cabo Verde. Entre los meses de mayo y junio caza ballenas en el océano que se extiende entre Cabo Verde y la isla de Trinidade. Permanece un par de días en Trinidade y sale rumbo a Río de Janeiro llegando el 31 de julio de $1790^{67}$, donde es visto el 6 de agosto ${ }^{68}$. El 16 de agosto zarpa con dirección a los mares del sur, siguiendo las huellas del Emelia ${ }^{69}$. Su paso por el cabo de Hornos no fue placentero y el escorbuto fue otro de sus problemas. En una carta enviada a los propietarios del buque señala: "tuve un impactante paso por el cabo de Hornos hacia afuera; estuve tratando de salir de ese lugar por casi dos meses antes de poder recorrerlo en uno de los climas más horribles que jamás barco u hombre ha experimentado; casi rompe en pedazos a mis hombres antes de llegar a la costa de Perú; [...] tuve siete hombres con escorbuto antes de abandonar la costa del Perú y si no hubiera sido por las calabazas que tenía cuando salí de la costa, habría tenido que enterrar la mitad de la tripulación del barco"70.

El Emelia sigue al Greenwich en lo que sería su segundo viaje al cabo de Hornos. Zarpa de Deal el 4 de julio de 1790 hacia las islas de Cabo Verde y luego se dirige a Río de Janeiro, desde donde sale hacia el mar del Sur el 6 de septiembre de

651 cwt [abreviación de 1 hundredweight] o quintal equivale a 112 libras (Zupko, 1985).

66 Ver https://whalinghistory.org/?s=BS0380.

67 Enderby, Samuel \& Alexander Champion, 1792.

68 Lloyd's List (Londres), 17 de noviembre de 1790.

69 Enderby, Samuel \& Alexander Champion, 1792.

70 [Captain Locke's Letter to Enderby \& Sons, Deal, 13 $3^{\text {th }}$ August 1791]. Enderby, Samuel \& Alexander Champion, 1792, f. 273.

71 Enderby, Samuel \& Alexander Champion, 1792.

72 [Captain Shields's Letter to Enderby \& Sons, St. Helena, $7^{\text {th }}$ July 1791]. Enderby, Samuel \& Alexander Champion, 1792, f. 274.

73 Enderby, Samuel \& Alexander Champion, 1792.
1790, luego de permanecer una semana en dicho puerto $^{71}$. No tenemos información sobre su paso por el cabo de Hornos, pero sí de sus problemas con el escorbuto. En una carta enviada desde la isla de Santa Helena el 7 de julio de 1791, el capitán Shields informa a los dueños del buque que "la mayor parte de mi tripulación tiene escorbuto y no seré capaz de salir de esta isla por algún corto tiempo"72.

Pocos días después, el 16 de julio de 1790, zarpa de Deal la fragata Atlantic, capitán Basset, también de Enderby \& Sons, rumbo a los mares del sur ${ }^{73}$. El buque había sido construido en Francia y tomado como presa en 1781 (Clayton, 2014, p. 63). Fue comprado en 1786 por S. Enderby \& Sons y registrado en Londres, con 237 toneladas, para destinarlo a la Southern Whale Fishery ${ }^{74}$. Toca en las islas de Cabo Verde, desde donde sale el 18 de agosto de 1790. Es visto a mediados de marzo de 1791 en el mar del Sur, con 75 toneladas de aceite $e^{75}$.

El Greenwich recala el 27 de mayo de 1791 en la isla Santa Helena, en su viaje de regreso. El Atlantic lo hace el 7 de julio y el Emelia el 21 de julio. Los buques estuvieron en faenas de cacería entre ocho y nueve meses ${ }^{76}$. El Greenwich llega a Gravesend el 15 de agosto ${ }^{77}$, el Emelia a Dover el 5 de septiembre ${ }^{78}$ y el Atlantic a Gravesend también el 5 de septiembre ${ }^{79}$.

El capitán Locke informa sobre la productividad del Greenwich y el Emelia: el capitán Shields y yo obtuvimos en compañía alrededor de 2000 barriles (250 toneladas) de aceite de esperma entre las latitudes 7 y $171 / 2$ sur a unas siete leguas de tierra firme ${ }^{80}$. El 15 de junio de 1791 el Emelia, capitán Shields, estaba en la isla Santa Helena con 168 toneladas de aceite $e^{81}$.

74 Register of Ships, 1786. En https://babel.hathitrust.org/ cgi/pt?id=hvd.32044020166658\&view=1up\&seq=147, consultado el 27 de abril de 2020.

75 Lloyd's List (Londres), 16 de agosto de 1791.

76 Enderby, Samuel \& Alexander Champion, 1792, fs. 273, 274.

77 Lloyd's List (Londres), 16 de agosto de 1791.

78 Enderby, Samuel \& Alexander Champion, 1792, f. 274.

79 Lloyd's List (Londres), 6 de septiembre de 1791.

80 [Captain Locke's Letter to Enderby \& Sons, Deal, 13 ${ }^{\text {th }}$ August 1791]. Enderby, Samuel \& Alexander Champion, 1792, f. 273.

81 Lloyd's List (Londres), 16 de agosto de 1791. 
El Greenwich regresa con 1.600 barriles, es decir, 200 toneladas $^{82}$, pero podría haber traído 50 toneladas más de lo que trajo sino se le hubiese enfermado parte de la tripulación con escorbuto ${ }^{83}$. Si sumamos las 200 del Greenwich con las $168 \mathrm{del}$ Emelia tenemos un total de 368 toneladas, más de las 250 que Locke indica habrían sido obtenidas en conjunto. La carga restante, 118 toneladas, pudo ser de aceite de ballena de barbas.

\section{CONCLUSIONES}

El viaje pionero del Emelia al océano Pacífico, rodeando en el mes de enero de 1789 el cabo de Hornos, abre una ruta segura que será navegada luego por cientos de buques balleneros provenientes de distintos puertos del hemisferio norte durante todo el siglo XIX.

En 1790 no solamente fueron los ocho balleneros ingleses que siguieron esa ruta $e$ ingresaron al océano Pacífico en busca de cachalotes. De Dunquerque, Francia, salieron siete balleneros: las fragatas Liberty, 490 ton, capitán Coleman; Necker, 300 ton, capitán Howes; Falkland, 180 ton, capitán Paddock; Penélope, 305 ton, capitán Worth; Bedford, 250 ton, capitán Coffin; y los bergantines Harmonie, 250 ton, capitán Starbuck; Marie, 240 ton, capitán Whippey (du Pasquier, 1990). Se informa, a comienzos de 1792, que "los siguientes buques pertenecientes a Dunkerque fueron avistados en buenas condiciones en la Pesca del Sur, alrededor del cabo de Hornos": Bedford, Coffin, Necker, Howes, Harmonie, Starbuck, y Marie, Whippey, el 25 de junio de 1791 y Penélope, Worth, y Liberty, Coleman, el 25 de julio ${ }^{84}$. Si sumamos todo lo producido por los balleneros de Dunquerque llegamos a los 5.480 barriles de aceite de cachalote (du Pasquier, 1990), con un promedio de 783 barriles por buque, es decir, cercano a las 100 toneladas ( 97,86 ton).

Desde los Estados Unidos los primeros buques rumbo al Pacífico recién salen en 1791: desde Nantucket, lo hacen las fragatas Rebecca, capitán Folger; Beaver, capitán Worth; Hector, capitán Brock, y Washington, capitán Bunker;

82 Un ton es igual a 8 barriles (Zupko, 1985).

83 Enderby, Samuel \& Alexander Champion, 1792, f. 274. y de New Bedford, la fragata Rebecca, capitán Kersey (Starbuck, 1878).

El marinero Elijah Durfey, a bordo del Rebecca, capitán Kersey, de New Bedford [17911793], cuenta que en 1792 había cuarenta buques balleneros en el Pacífico: veintidos de Inglaterra, ocho de Dunkerque, siete de Nantucket $y$ uno de New Bedford, de Hudson y de Boston (Stackpole, 1972, p. 130). La información es recogida por Eugenio Pereira Salas, le agrega una lista con los nombres de los buques y señala que la mayor parte volvió a sus puertos de origen con cargamentos completos; algunos pasaron a ser arquetipos de esta forma de empresa y dejaron su nombre inscrito en los anales de la historia de la pesquería (Pereira Salas, 1971, p. 44).

El cachalote capturado el 3 de marzo de 1789 frente a Tongoy por el Emelia fue el primero de una larga serie de ejemplares cazados en las costas de Chile. En Magallanes la actividad ballenera fue realizada durante gran parte del siglo XIX por naves extranjeras (algunas embarcaciones chilenas, en el fin-de-siécle, se aventuraron también pero sólo en la caza de lobos). La ruta del estrecho de Magallanes, además de la del cabo de Hornos, fue ocupada igualmente por buques balleneros y loberos para llegar a los caladeros del Pacífico a partir de la década de 1820 (Mayorga, 2020).

El verdadero auge de la caza de ballenas en los mares australes surge recién en la primera década del siglo XX cuando empresarios magallánicos se unen con especialistas noruegos para desarrollar una caza moderna de ballenas, que los llevará no sólo a recorrer el estrecho de Magallanes, los canales patagónicos occidentales, sino también las aguas antárticas en busca de los apreciados cetáceos. La caza de ballenas se mantuvo por casi dos siglos en Chile, finalizando recién en 1983 (Quiroz, 2020).

\section{AGRADECIMIENTOS}

Nuestra gratitud al Programa Fondecyt por el financiamiento otorgado al Proyecto Regular 1170318 "Narrativas etnográficas y operaciones balleneras en las costas sudamericanas entre los 
siglos XVII y XX: patrones, transformaciones y continuidades", marco en el que este trabajo fue escrito; a los funcionarios de la Caird Library and Archive del National Maritime Museum, de la British Library y del The National Archives, instituciones todas de Londres, por las facilidades para consultar y fotografiar los ejemplares de la carta del capitán Shields a Samuel Enderby \& Sons, que nos motivó a escribir este trabajo; a mi hija Daniela Quiroz por su claridad para poner en un mapa lo que el escrito necesitaba; y a los dos revisores del artículo, pues sus recomendaciones permitieron mejorar el texto de manera significativa.

\section{FUENTES DE CONSULTA}

\section{British Library, Londres, Inglaterra}

An account of the vessels now out on the Southern Whale Fishing made up to $31^{\text {st }}$ December 1778 from Enderby \& Sons. Liverpool Papers, Volumen I (Add MS 38391 [1788-1789), fs. 92-93.

[Declaración de Samuel Enderby ante el Committee of Trade \& Foreign Plantation, 17 de enero de 1791]. Serie Papers of Charles Jenkinson, $1^{\text {st }}$ Earl of Liverpool, Subseries Add. MS 38393, ff. 10-11.

Letter from Samuel Enderby on first whaling vessel to round Cape Horn into South Sea. Serie Papers of Charles Jenkinson, $1^{\text {st }}$ Earl of Liverpool, Subseries Add. MS 38388-38394, f. 71.

Letter of Captain James Shields, Master of the ship Emilia to S Enderby and Sons, 1790 (copy). Liverpool Papers, Volumen I (Add MS 38225 [Jan 1790-Nov 1790]), fs. 70-71.

Question to be submitted to Enderby \& Champion on Southern Whale Fishery, March $10^{\text {th }} 1792$. Liverpool Papers, Volumen I (Add MS 38393, [6 Jan 1791-31 Mar 1792]), f. 198.

Reply by whaling merchants to questions about Southern Whale Fishery. Liverpool Papers, Volumen I (Add MS 38393, [6 Jan 1791-31 Mar 1792]), fs. 205-206.

\section{London Metropolitan Archives, Londres, Inglaterra}

Insurance Ship Emelia (James Shields commander). Collection Royal \& Sun Alliance Insurance Corporation, CLC/ B/192/F/001/MS11936/378/58871.

The National Archives, Kew, Londres, Inglaterra
An account of Number of Ships with their Cargoes, which returns from the Southern Whale Fishery to England in the year 1788, as furnished by Mr. Enderby, one of the Adventurers. Public Record Office, PRO 30/8/259, f. 160.

An account of the Number of Ships, and their Tonnage, fitted out in Great Britain for the Southern Whale Fishery, 10 de abril de 1786. Public Record Office, PRO 30/8/259, f. 199.

A State of the Southern Whale Fishery, 16 de mayo de 1790. Public Record Office, PRO 30/8/259, f. 161.

Commissioners Customers on Memorial of Samuel Enderby \& Premium on 5 ships employed in the Southern Whale Fishery. Records of Treasury, T1/671, item 1566.

Council recommending a stay of process against some spirits belonging to Messrs. Enderby \& Sons returned in the ship Emilia from the Southern Whale Fishery. Public Record Office, Treasury, T1/682/1369, fs. 293295.

Letter of Samuel Enderby Jr. a William Pitt Jr. [Statement on Southern Whale Fishery], 30 de agosto de 1790. Public Record Office, PRO 30/8/133, fs. 35-36.

Letter of Samuel Enderby Jr. to Evan Nepean, 16 de agosto de 1790. Home Office 42/16, Letters and papers, 1790 Jan 01-1790 to Aug 31, fs. 288-292.

Particulars relating to Every English Vessel which has gone into and returned from the Whale Fishery in the Pacific Ocean, 1792 by Samuel Enderby \& Alexander Champion. Records on the Board and Trade, BT 1/2 part 2, (October 1791-March 1792) fs. $271-274$.

\section{National Maritime Museum, Greenwich, Londres, Inglaterra}

James Shields to Saml. Enderby \& Sons, 6 March 1790 (copy, 2 pages). Markham Family Papers, MRK/105/1/113; Caird Library \& Archive.

National Library of Australia, Canberra, Australia

Letter of Captain J. Shields to Samuel Enderby and Sons, 06 March 1790, https://nla.gov.au/nla.obj-761982653/ view, consultado el 28 de marzo de 2020.

Letter of Samuel Enderby to Evan Nepean, enclosing copy of letter from Captain Shields of voyage of Emilia in South Seas (6 March 1790), https://nla.gov.au/nla.obj-728094411/ view, consultado el 28 de marzo de 2020. 


\section{Periódicos}

Caledonian Mercury (Edimburgo), 1790.

Hampshire Chronicle (Hampshire), 1790.

Hereford Journal (Hereford), 1790.

Hull Packet, The (Hull), 16 de mayo de 1809.

Friend, The (Honolulu), $1^{\circ}$ de mayo de 1872.

Lloyd's List (Londres), 1788-1797.

York Herald, The (York), 5 de julio de 1823.

\section{Bases de Datos Online}

Register of Ships, 1782. En https://babel.hathitrust.org/cgi/ pt?id=hvd $.32044019372358 \& v i e w=1$ up\&seq $=98$, consultado el 26 de abril de 2020.

Register of Ships, 1783. En https://babel.hathitrust.org/cgi/ pt?id=hvd $.32044020166674 \&$ view $=1$ up\&seq $=99$, consultado el 26 de abril de 2020.

Register of Ships, 1786. En https://babel.hathitrust.org/cgi/ pt?id=hvd. $32044020166658 \&$ view $=1$ up\&seq $=104$, consultado el 26 de abril de 2020.

Register of Ships, 1789. En https://babel.hathitrust.org/cgi/ pt id $=$ hvd .32044020166310 \&view $=1$ up\&seq $=5$, consultado el 31 de marzo de 2020.

Register of Ships, 1800. En https://books.google.cl/ books?id=v6yaIbUX6IMC\&pg =PP1\&redir esc $=\mathrm{y}_{\mathrm{V}}=$ onepage $\& \mathrm{q} \& \mathrm{f}=$ false, consultado el 31 de marzo de 2020.

https://whalinghistory.org/?s=BS0303, consultado el 31 de marzo de 2020.

https://whalinghistory.org/?s=BS0380, consultado el 31 de marzo de 2020.

https://whalinghistory.org/?s=BV030300, consultado el 31 de marzo de 2020.

http://www.lynnesgenealogy.com/HammondDescendants/ website/b3166.htm\#P3171, consultado el 31 de marzo de 2020.

http://www.spanglefish.com/slavesandhighlanders/index. asp? pageid $=670520$, consultado el 30 de marzo de 2020.

https://www.bankofengland.co.uk/monetary-policy/inflation/ inflation-calculator, consultado el 10 de octubre de 2020 .

\section{BIBLIOGRAFÍA}

Alston, D. (n/d). Slaves \& Highlanders. En http://www. spanglefish.com/slavesandhighlanders/index.asp; consultado el 25 de marzo de 2020.
Andress, D. (2008). 1789: The Threshold of the Modern Age. New York: Farrar, Straus and Giroux.

Beale, Th. (1839). The Natural History of the Sperm Whale, to which is added a sketch of a South-sea whaling voyage. Londres: John van Voorst.

Bennett, F. D. (1840). Narrative of a whaling voyage round the globe from the year 1833 to 1836. Londres: Richard Bentley.

Briggs, L. V. (1889). History of Shipbuilding on North River. Boston: Coburn Brothers.

Clark, H. (1887). The Whale Fishery. In G. B. Goode (Ed.), The Fisheries and Fishery Industries of the United States. Sección V, Volumen II, pp. 3-218. Washington: Government Office Printing.

Clayton, J. (2014). Ships Employed in the South Sea Whale Fishery from Britain: 1775-1815. Hertfordshire: Berforts Information Press.

Convención de Nootka (1796). Convención concluida entre España e Inglaterra, transigiendo varios puntos sobre pesca, navegación y comercio en el Océano Pacífico y los mares del Sur, firmada en San Lorenzo el Real a 28 de octubre de 1790. En Colección de los Tratados de Paz, Alianza \&, Comercio, ajustados por la Corona de España con las Potencias Extranjeras desde el Reynado del Señor Felipe Quinto hasta el Presente, Tomo I: 365-372. Madrid: Imprenta Real.

Croix, T. de (1859 [1790]). Memoria de los Virreyes que han gobernado el Perú durante el tiempo del Coloniaje español. Tomo V. Lima: Librería Central de Felipe Bailly.

Dakin, W. J. (1938). Whalemen adventures. Sydney: Angus \& Robertson.

Daughan, G. C. (2013). The Shining Sea: David Porter and the Epic Voyage of the U.S.S. Essex during the war of 1812. New York: Basic Books.

Dawson, K. R. (2010). Caroline, Princess of Wales \& other forgotten people of the History. Durham: Eloquent Books.

Dolin, E. J. (2007). Leviathan. The history of Whaling in America. New York: Norton \& Co.

Ellis, R. (2011). The Great Sperm Whale. Lawrence: University Press of Kansas.

Enderby, Ch. (1848). Proposal for the re-establishing the British Southern Whale Fishery. Londres: Royal Exchange.

Essex Institute. (1906). Ship Register of the district of Salem \& Beverly, 1789-1900. Salem: Essex Institute.

Francis, D. (1990). A history of world whaling. Markham: Penguin Books. 
Fraser, C. (2014). The Enderby settlement. Otago: Otago University Press.

Hamilton, D. (2015). Scotland, the Caribbean and the Atlantic world, 1750-1820. Manchester: Manchester University Press.

Holt, F. L. (1820). A System of Shipping and Navigation Laws of Great Britain and of the Laws relative to Merchant Ships and Seamen and Maritime Contracts. Londres: Joseph Butterworth \& Sons.

Jackson, G. (1978). The British Whaling Trade. Hamden: Archon Books.

Jones, A. (1981a). The British Southern Whale and Seal Fisheries. The Great Circle, 3(1), 20-29.

Jones, A. (1981b). The British Southern Whale and Seal Fisheries. Part II: the principal operators. The Great Circle, 3(2), 90-102.

Jones, A. (1991). Ships employed in the South Seas Trade, 1775-1859. Roebuck Society Publications $N^{\circ} 16$. Burwood (VA): The Australian Association for Maritime History Inc.

Kirk, R. W. (2012). The Lost Paradise: the transformation of South Pacific, 1520-1920. Jefferson, NC: McFarland $\&$ Co.

Macy, O. (1835). The history of Nantucket. Boston: Hillard, Gray \& Co.

Mawer, G. A. (1999). Ahab's Trade. The saga of South Seas Whaling. New York: St. Martin's Press.

Mayorga, M. (2020). Pieles, tabaco y quillangos. Relaciones entre loberos estadounidenses y aborígenes australes en la Patagonia (1780-1850). Santiago: Servicio Nacional del Patrimonio Cultural.

Melville, H. (2002 [1851]). Moby Dick. New York: Norton \& Co.

Nichols, P. (2009). Oil \& Ice. A Story of Arctic Disaster and the Rise and Fall of America's Last Whaling Dynasty. New York: Penguin Books.

Ortiz Sotelo, J. (2015). La Real Armada en el Pacífico Sur: el Apostadero Naval del Callao 1746-1824. México: Universidad Nacional Autónoma de México.

Pasquier, Th. du. (1990). Les baleiniers français de Louis XVI a Napoléon. Paris: Henri Veyrier.

Payton, Ch. (2018). The Enderby family and their world. In Ch. Elmers \& Ch. Payton (Eds.), London and the whaling trade (pp. 185-210). Londres: Lavenham Press Ltd.

Pereira Salas, E. (1971). Los primeros contactos entre Chile y los Estados Unidos, 1778-1809. Santiago: Andrés Bello.

Philbrick, N. (2011). Away of Shore. Nantucket Island and its people, 1602-1890. New York: Penguin Books.
Quiroz, D. (2020). Soplan las ballenas. Historias sobre la caza de cetáceos en las costas de Chile. Santiago: Centro de Investigaciones Diego Barros Arana.

Rediker, M. (2007). The Slave Ship: a Human History. Londres: Viking Penguin Books.

Richardson, D. (1997). Bristol, Africa and the Eighteenth Century Slave Trade to America: Volume 4, The Final Years, 1770-1807. Bristol: Bristol Record Society Publications.

Richmond, Th. (1868). The local records of Stockton and the Neighbourhood. Stockton: William Robinson.

Russell, F. (1786). A Collection of Statutes Concerning the Incorporation, Trade, and Commerce of the East Indian Company \& the Government of the British Possessions in India. Londres: Charles Eyre \& Andrew Strahan.

Sanderson, I. T. (1993). A History of Whaling. NewYork: Barnes \& Noble.

Sanford, F. C. (1872). Notice of Early Nantucket whalemen. Proceedings of American Antiquarian Society, 1872: 28-29.

Sanford, F.C. (1884). Notes upon the history of American Whale Fishery. In Report of the Commissioner of Fish and Fisheries for 1882 (pp. 205-220). Washington: Government Printing Office.

Scammon, Ch. M. (1874). The marine mammals of the north-western Coast of North America, described and illustrated, together with an account of the American Whale Fishery. San Francisco: John H. Carmany \& Co.

Shore, C. M. (2012). "Does the whale's magnitude diminishes?". Melville's use of Thomas Beale's The Natural History of Sperm Whales. Thesis of Master of Arts, English Department, Appalachian State University, Boone NC.

Slevin, J. R. (1959). The Galapagos Islands. A history of their exploration. San Francisco: California Academy of Sciences.

Spate, O. H. K. (1979). The Spanish Lake. Canberra: Australian National University Press.

Spears, J. (1908). The story of the New England whalers. New York: The Macmillan Co.

Spence, B. (1980). Harpooned. The story of whaling. Greenwich, Londres: Conway Maritime Press.

Stackpole, E. (1972). Whales and Destiny. The rivalry between America, France, and Britain for control to the Southern Whale Fishery, 1785-1825. Amherst: University of Massachusetts Press.

Stackpole, E., \& Summerfield, M. (1974). Nantucket 
Doorways. Tresholds to the Past. New York: Hastings House.

Starbuck, A. (1878). History of American Whale Fishery from its earliest inception to the year 1876. Washington: Government Printing Office.

Starkey, D. J. (1990). British Privateering Enterprise in the Eighteenth Century. Exeter: University of Exeter Press.

Valdés Hansen, F. (2014). Balleneros del norte rumbo al Sur: las primeras industrias en Sudamérica. En D. Quiroz
\& P. Toledo (Eds.), Balleneros del Sur. Antropología e Historia de la industria ballenera en las costas sudamericanas (pp. 17-54). Santiago: Andros.

Webb, R. L. (1988). On the Northwest. Commercial whaling in the Pacific Northwest (1790-1967). Vancouver: University of British Columbia.

Zupko, R. E. (1985). A dictionary of weights and measures for the British Isles: The Middle Ages to the Twentieth Century. Philadelphia: American Philosophical Society. 